\title{
Complications and Toxicities Associated with Cancer Therapies in the Intensive Care Unit
}

\author{
Melvin J. Rivera, Bryan Do, Jeffrey C. Bryan, Terri Lynn Shigle, \\ and Rina Patel
}

\section{Contents}

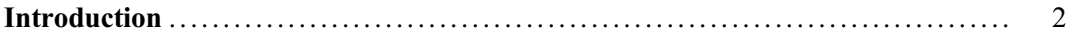

Toxicities of Anticancer Therapy $\ldots \ldots \ldots \ldots \ldots \ldots \ldots \ldots \ldots \ldots \ldots \ldots \ldots \ldots \ldots \ldots \ldots \ldots \ldots \ldots, 2$

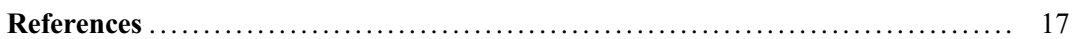

M. J. Rivera

Clinical Pharmacy Specialist - Thoracic / Head and Neck Medicine, The University of Texas MD Anderson Cancer Center, Houston, TX, USA

e-mail: mjrivera@mdanderson.org

B. Do

Clinical Pharmacy Specialist - Lymphoma and Myeloma, The University of Texas MD Anderson Cancer Center, Houston, TX, USA

e-mail: bdo@mdanderson.org

J. C. Bryan

Clinical Pharmacy Specialist - Leukemia, The University of Texas MD Anderson Cancer Center, Houston, TX, USA e-mail: jcbryan@mdanderson.org

\section{T. L. Shigle}

Clinical Pharmacy Specialist - Stem Cell Transplantation and Cellular Therapy, The University of Texas MD

Anderson Cancer Center, Houston, TX, USA

e-mail: tshigle@mdanderson.org

\section{R. Patel $(\bowtie)$}

Clinical Pharmacy Specialist - Critical Care / Nutrition

Support, The University of Texas MD Anderson Cancer

Center, Houston, TX, USA

e-mail: rppatel1@mdanderson.org

\section{Abstract}

Advances in the management of hematologic malignancies and solid tumors have given rise to diverse modalities to treat cancer other than cytotoxic chemotherapy, including targeted therapies, immunotherapies, and cellular therapies. Currently, there are over 175 FDA-approved antineoplastic agents in the United States, many with a diverse and profound toxicity profile. Complications of antineoplastic therapy may result in the need for intensive care unit (ICU) admission to provide acute symptom management. Accordingly, ICU providers caring for cancer patients should have a working knowledge of the toxicities and complications associated with antineoplastic therapy.

\section{Keywords}

Chemotherapy $\cdot$ Immunotherapy $\cdot$ Cancer $\cdot$ Toxicity $\cdot$ Oncology $\cdot$ Critical care $\cdot$ Intensive care $\cdot$ Complications $\cdot$ Adverse effects . Antineoplastic agents 


\section{Introduction}

The prevalence of cancer has grown tremendously and with that so has the need for new or repurposed anticancer therapies. Advances in the management of hematologic malignancies and solid tumors have given rise to diverse modalities to treat cancer other than cytotoxic chemotherapy, including targeted therapies, immunotherapies, and cellular therapies. There are over 175 approved antineoplastic agents in the United States and more in development with unique toxicity profiles [163]. This has created a unique opportunity for critical care specialists to manage complications of critically ill cancer patients receiving anticancer therapies.

\section{Toxicities of Anticancer Therapy}

Tables $1 \mathrm{~A}-\mathrm{H}$ provides a list of antineoplastic agents and toxicities that may necessitate a higher level of care and/or impact care within the intensive care unit (ICU). These tables are not all-inclusive of every minor adverse effect of each agent. Instead, they focus on toxicities that are considered severe/ life-threatening complications or clinically relevant (e.g., grade 3 or 4 adverse effects). Agents that did not meet the criteria for addition to Tables $1 \mathrm{~A}-\mathrm{H}$ include the following: azacitidine, cladarabine, decitabine, elotuzumab, hydroxyurea, ixazomib, lomustine, olaratumab, omacetaxine, procarbazine, talimogene, valrubicin, and venetoclax. 


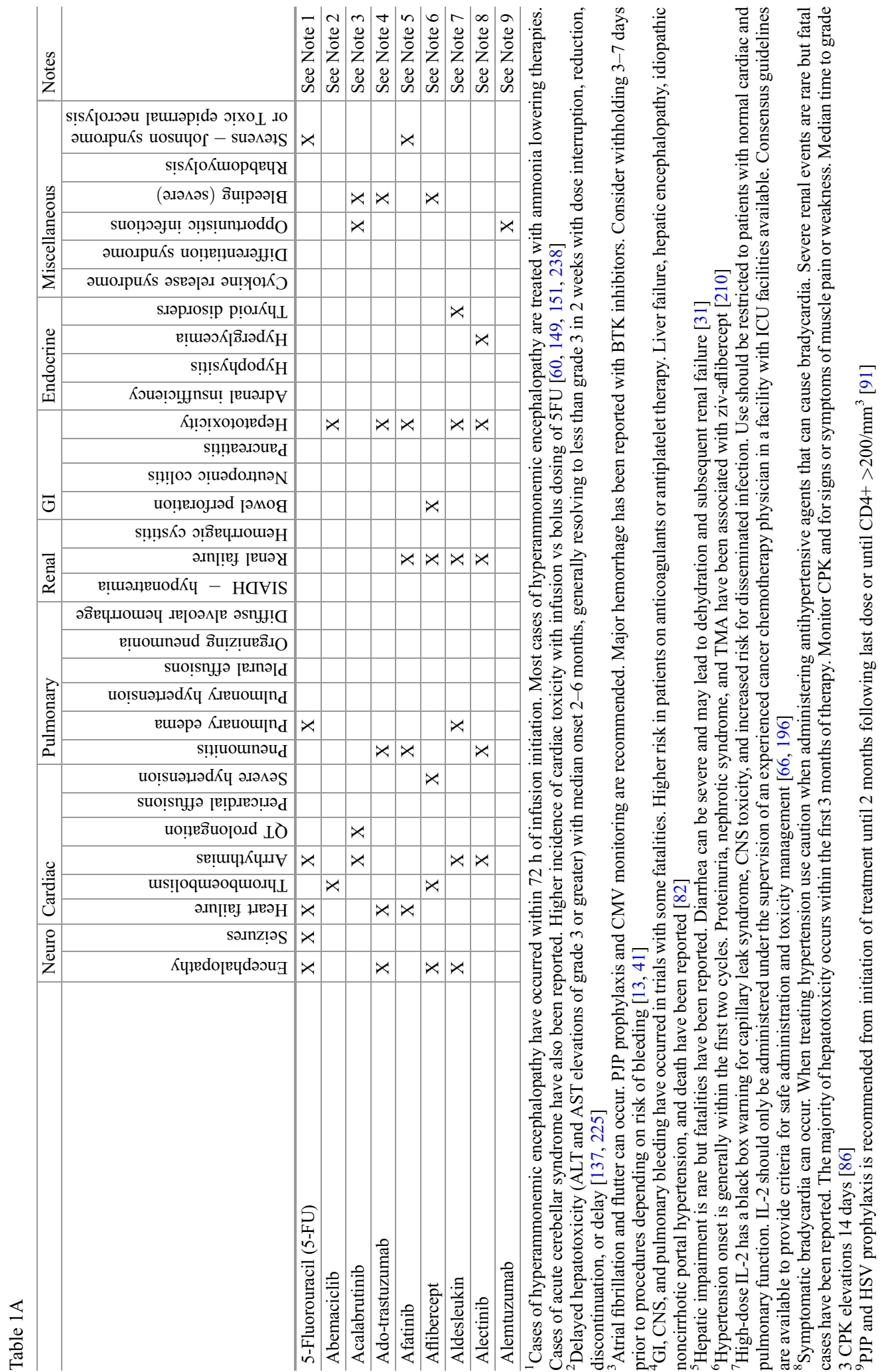




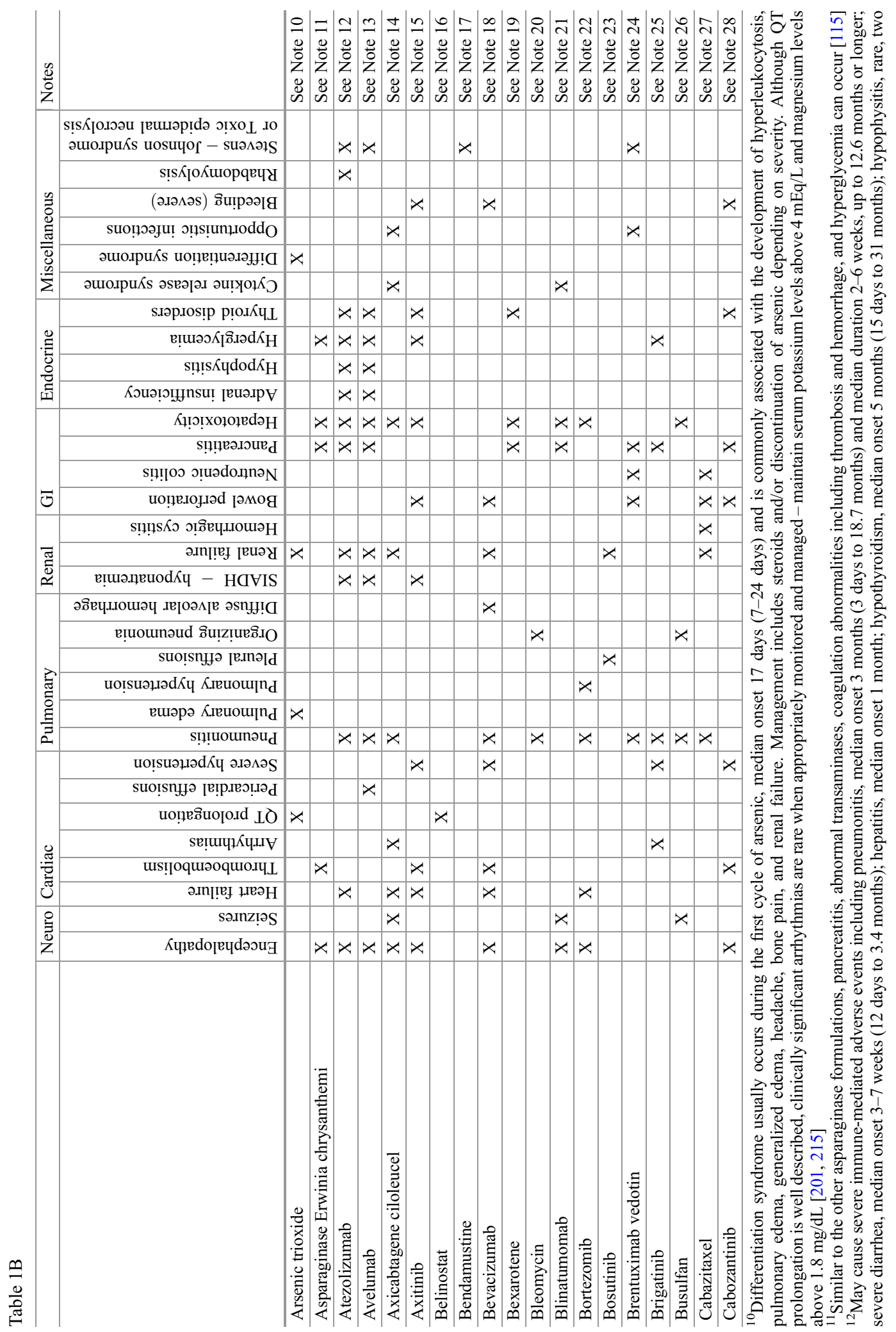




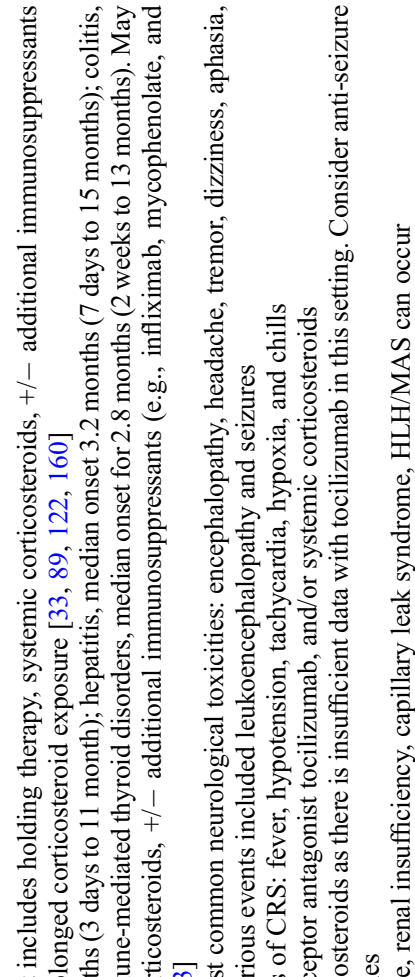

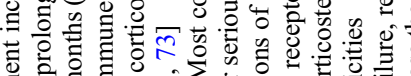

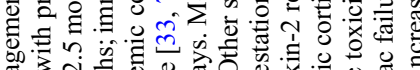

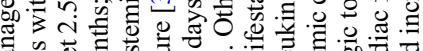

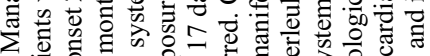

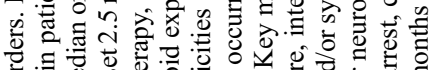

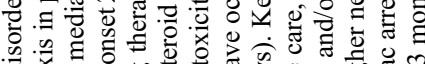

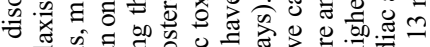

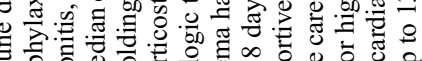

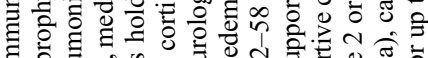

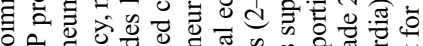

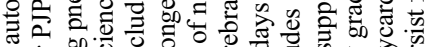
on

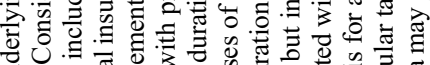

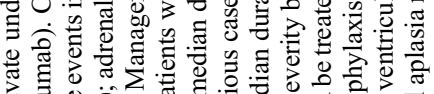

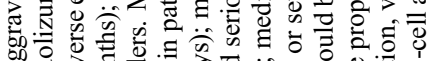

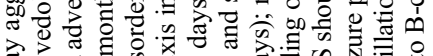

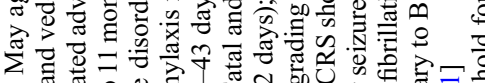

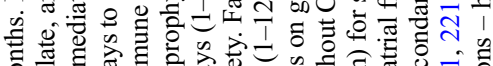

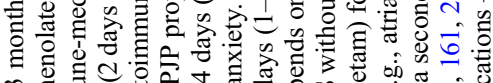

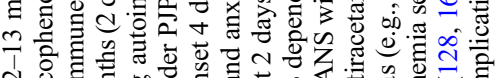

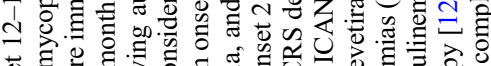

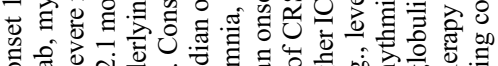

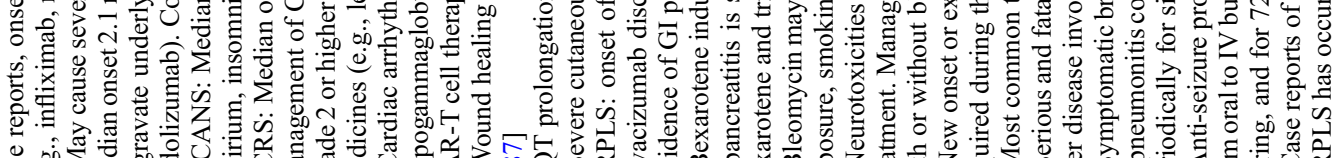

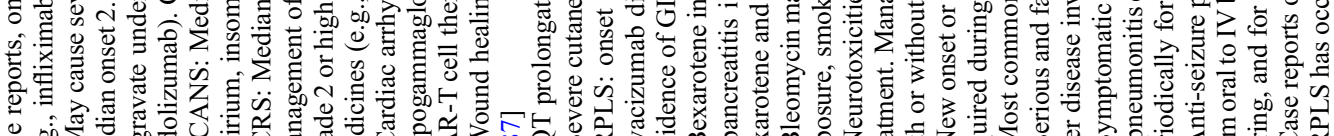

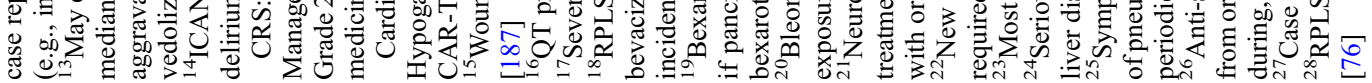




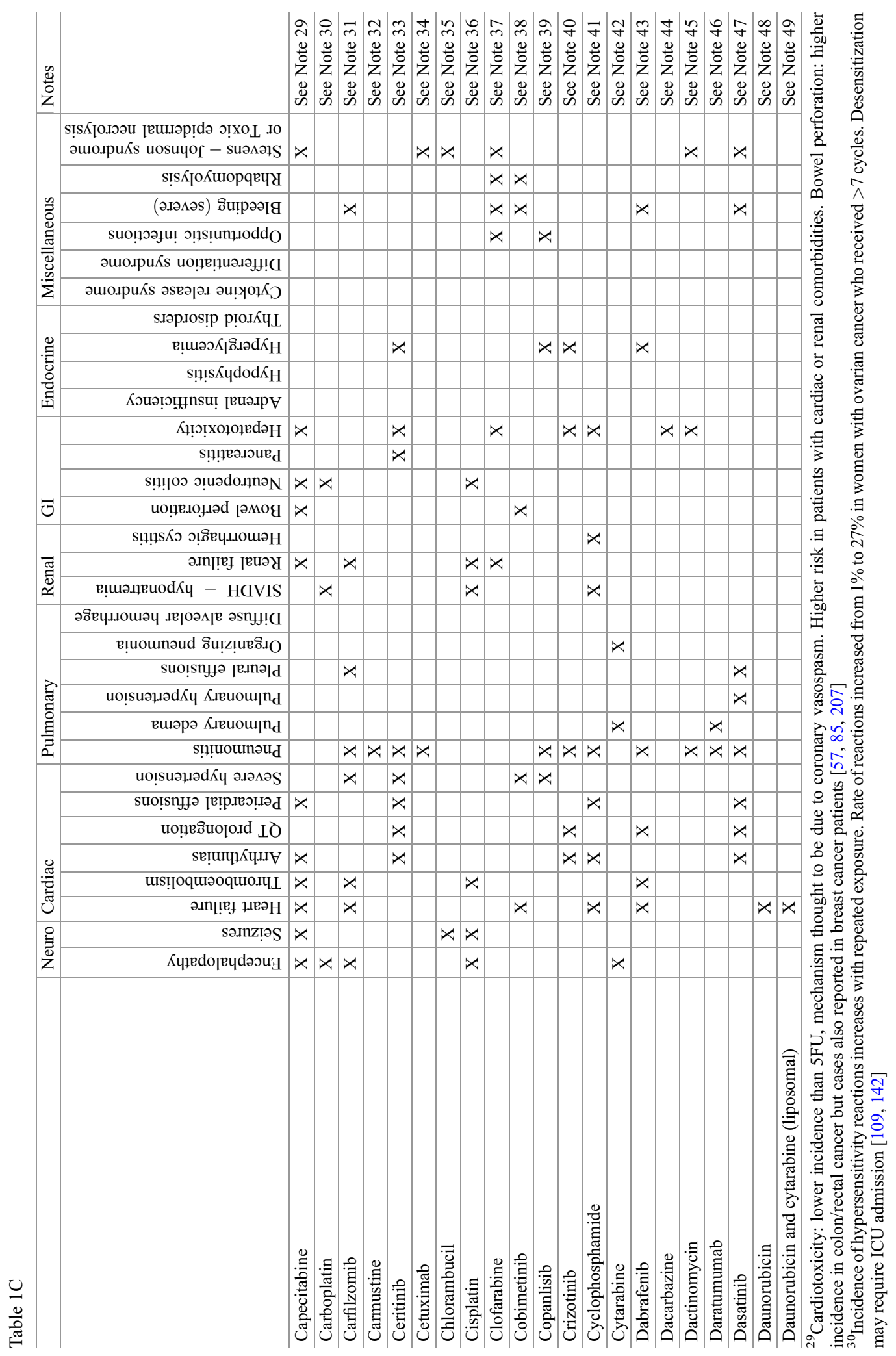




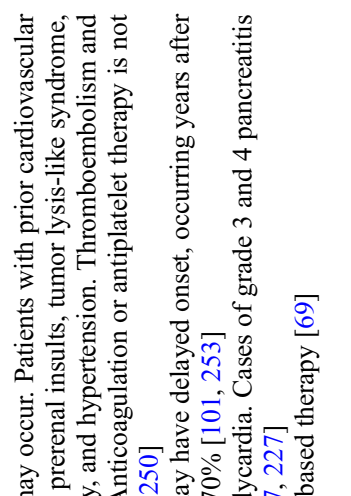

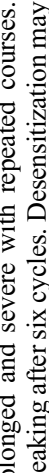

更

ô

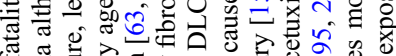

on

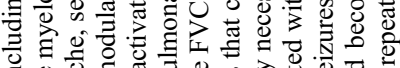

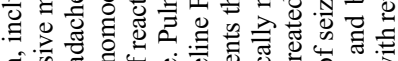

50

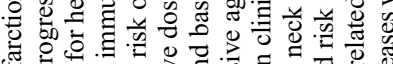

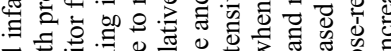

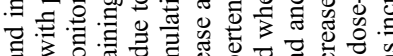

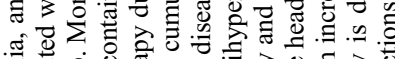

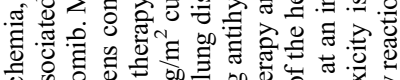

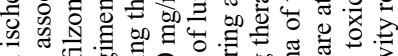

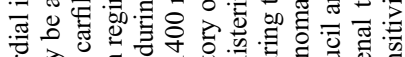

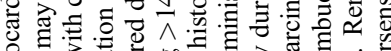

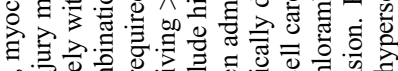

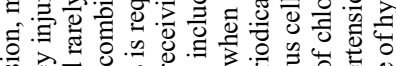

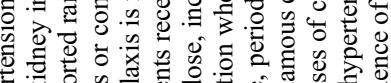

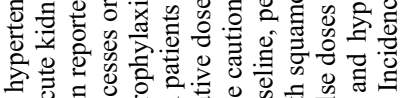

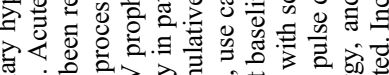

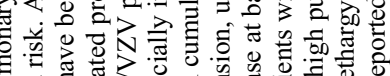

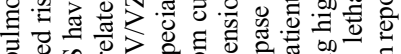

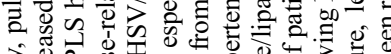

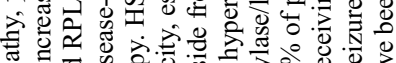

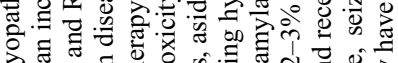

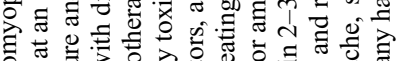

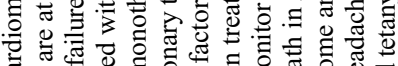

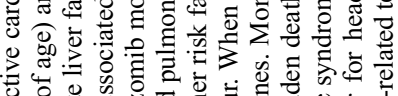

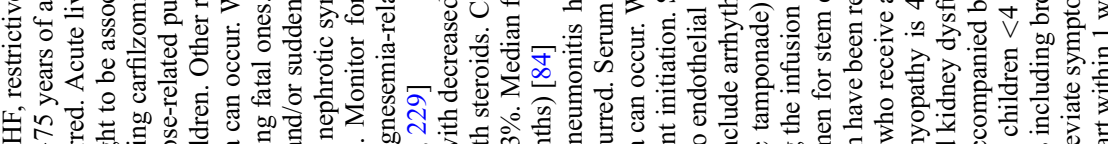
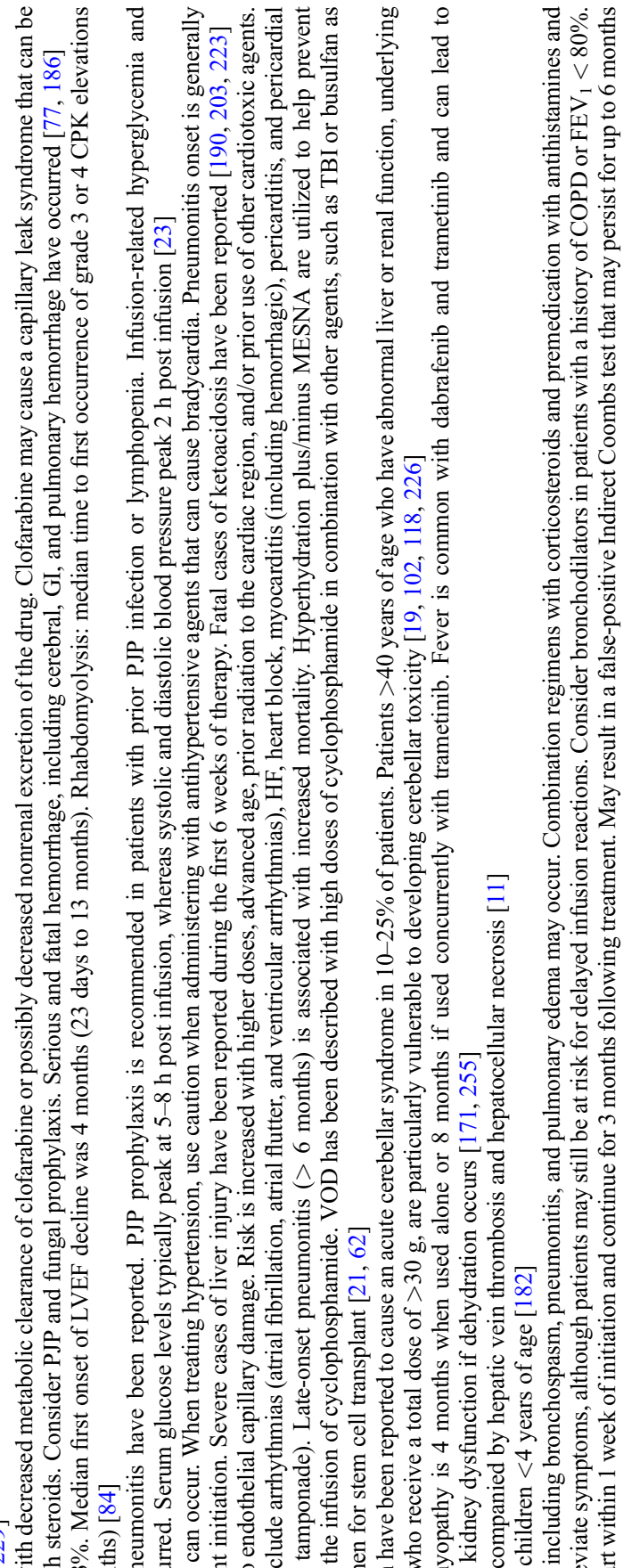

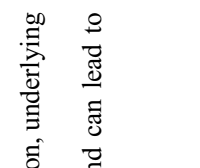

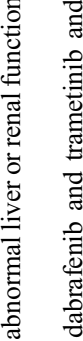

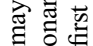

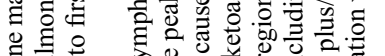

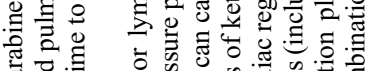

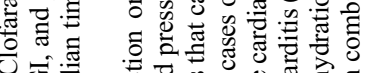

U.

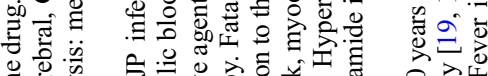

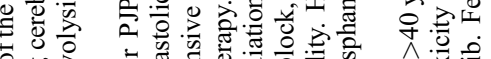

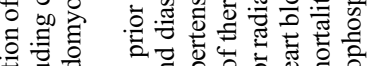

里的

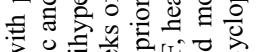

离 竞

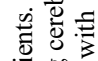

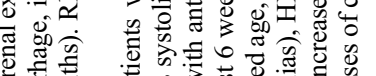

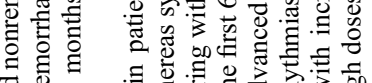

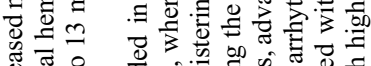

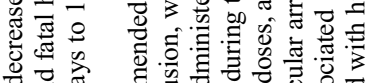

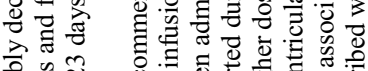

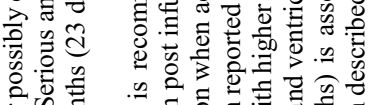

के

艾

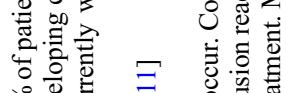

胫言

응 :

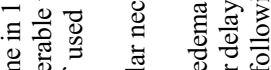

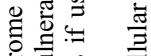

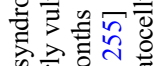

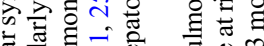

ప

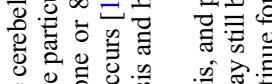

๘ँّ

응 号员

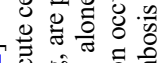

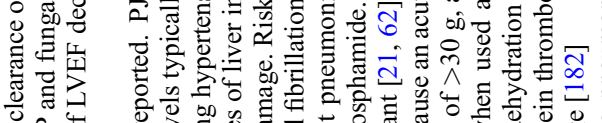

릉

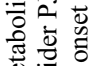

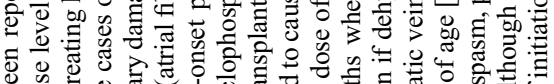

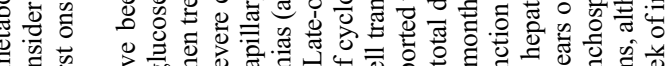

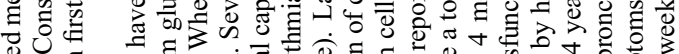

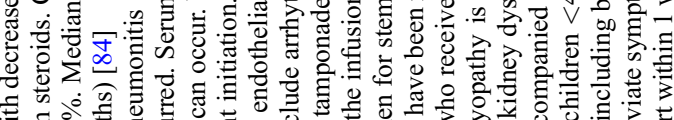

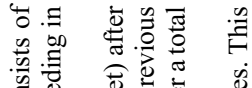

ठ․ㅠ

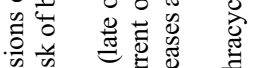

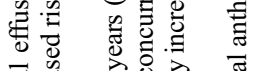

त्ञّ

ป

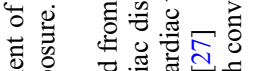

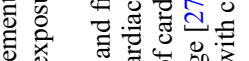

(⿻)

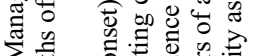

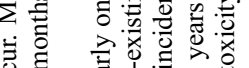

घ हों

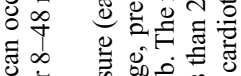

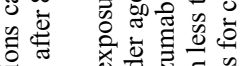

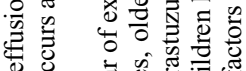

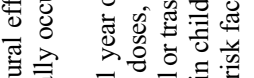

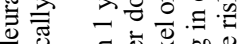

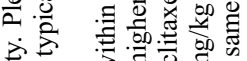

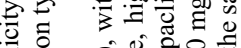

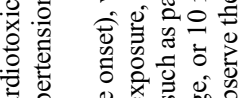

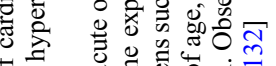

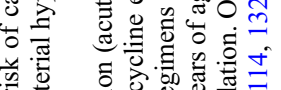

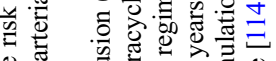

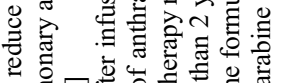

열 可论

ते

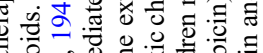

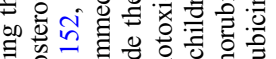

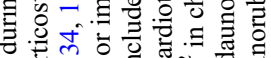

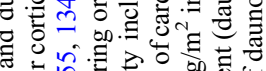

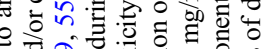

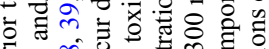

है

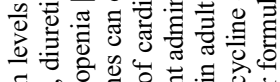

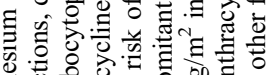

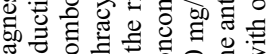

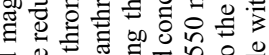
च

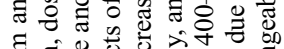

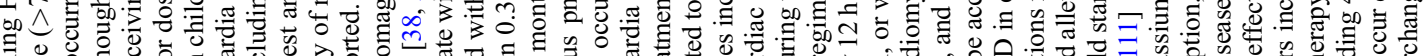
吾。

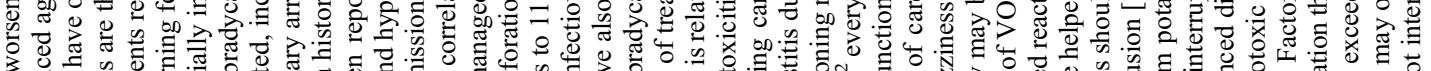

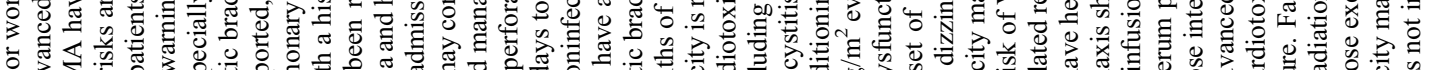

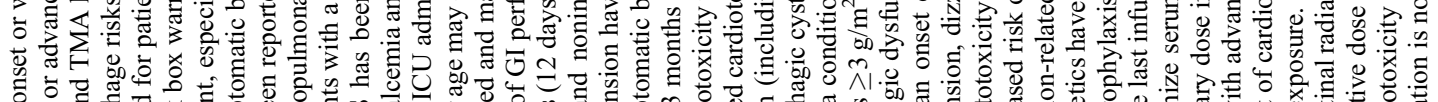

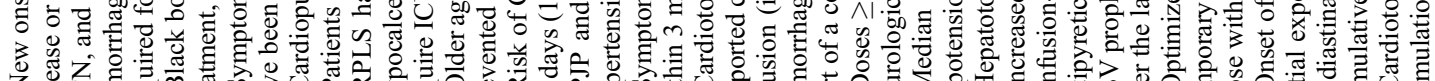

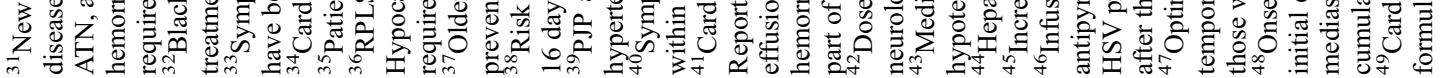









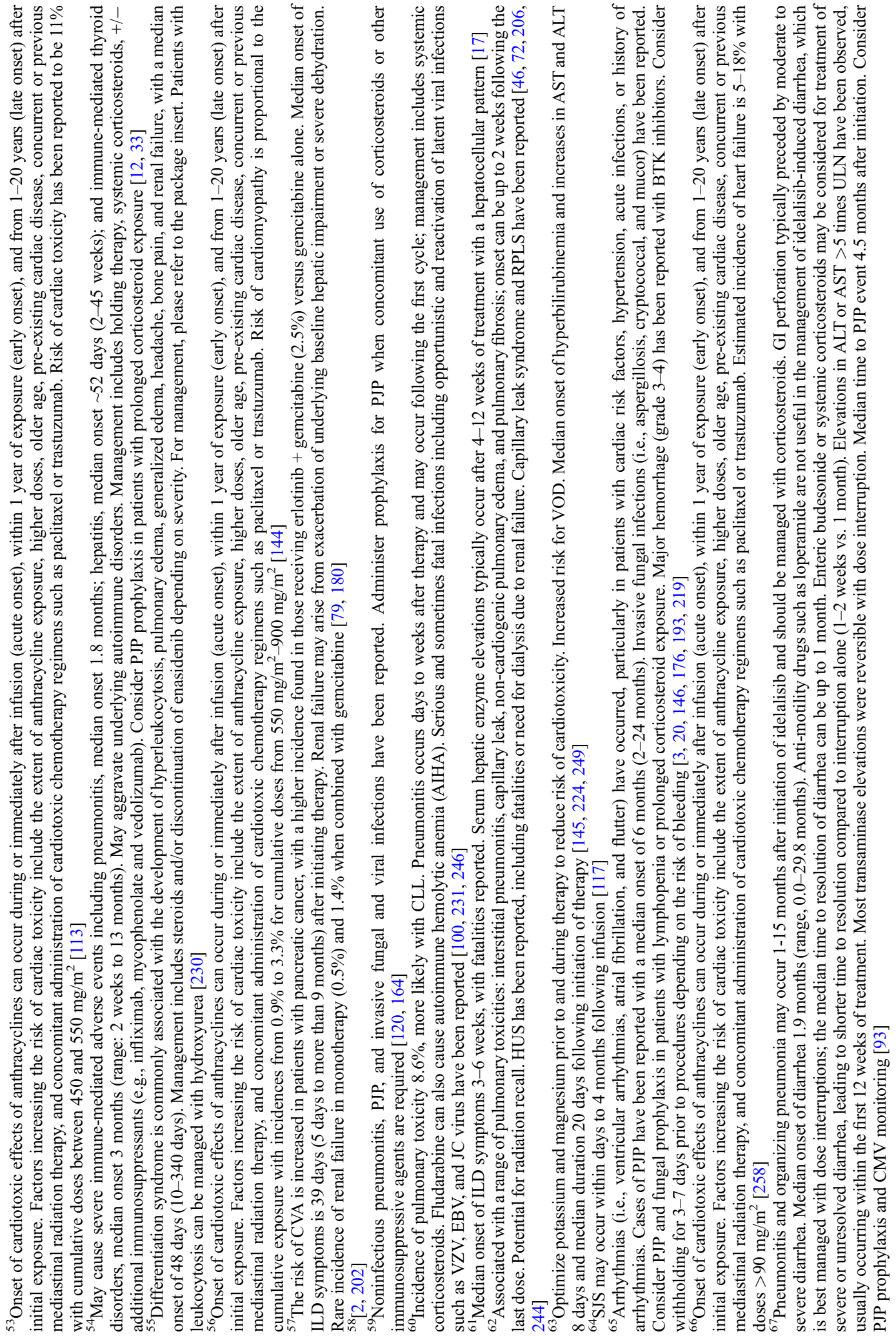




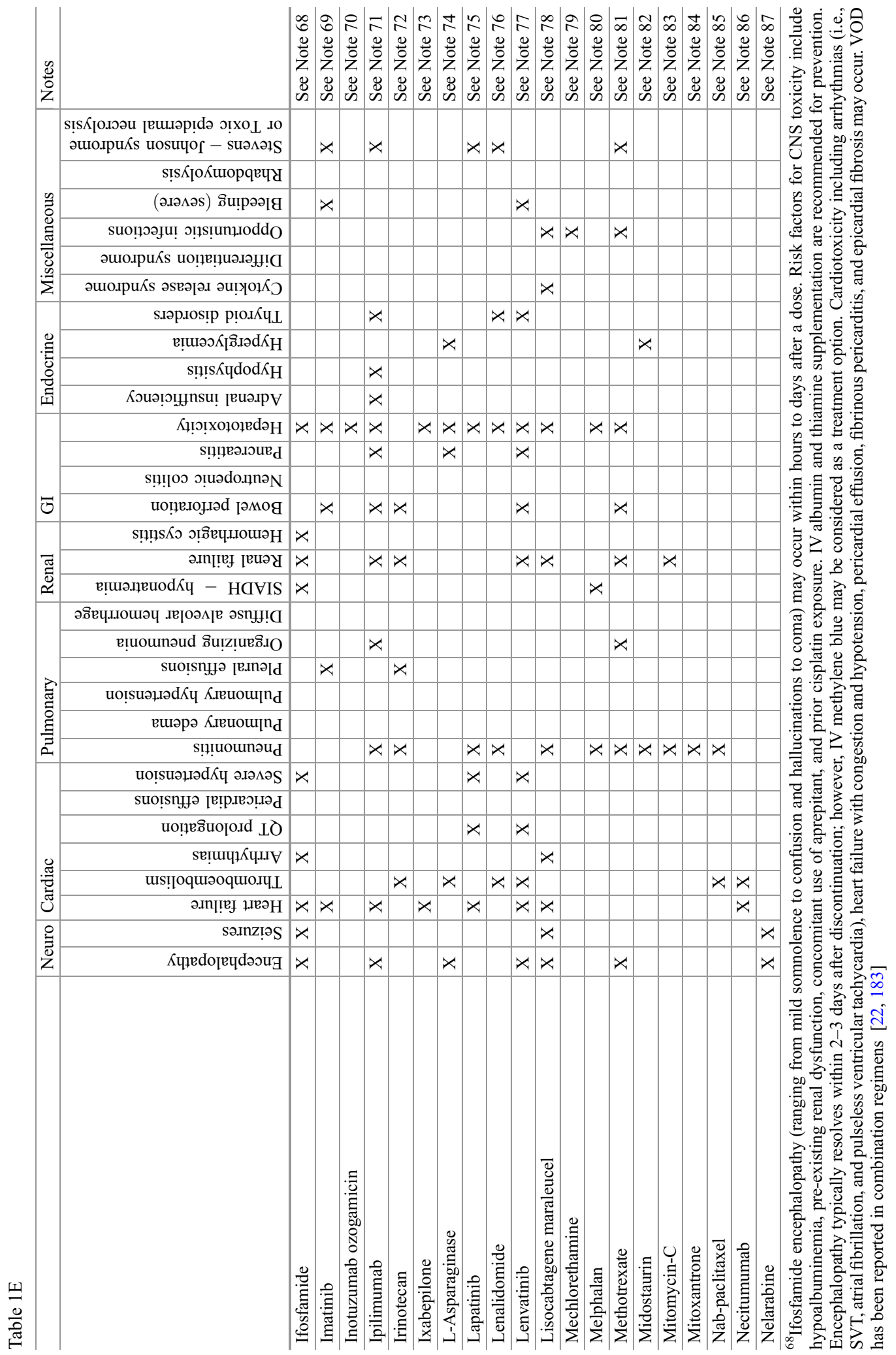




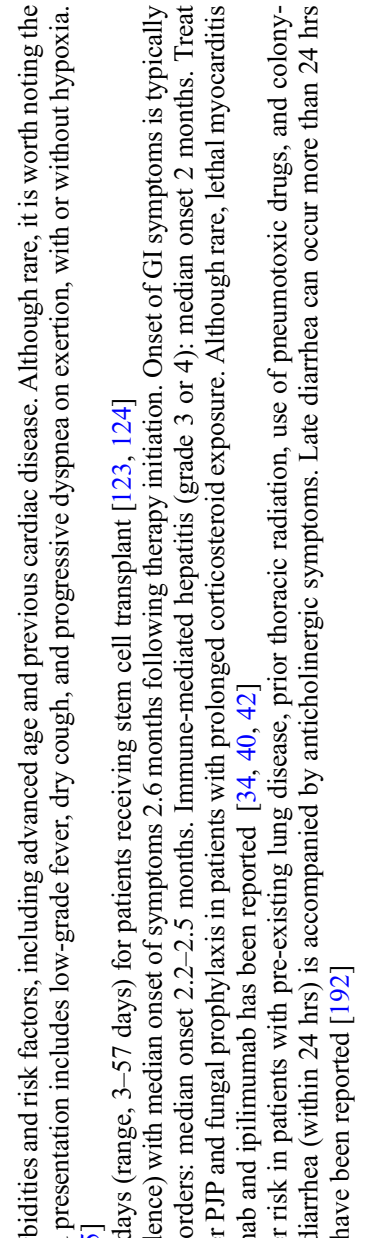

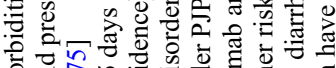

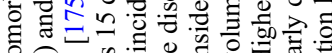

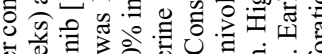

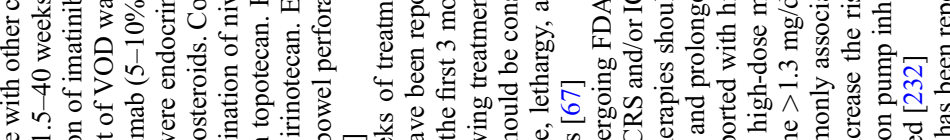

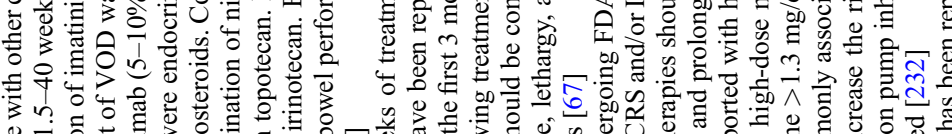

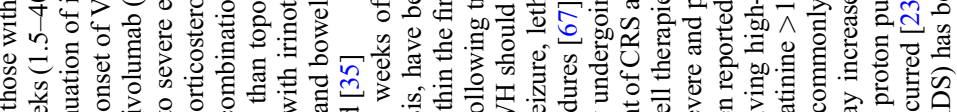

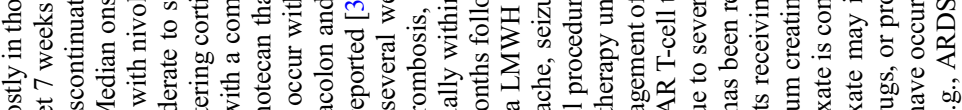

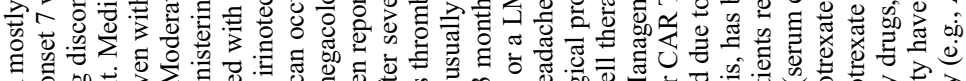

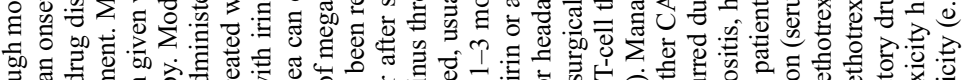

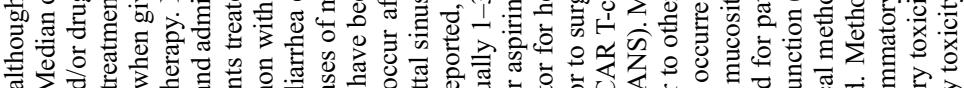

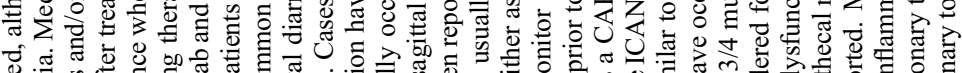

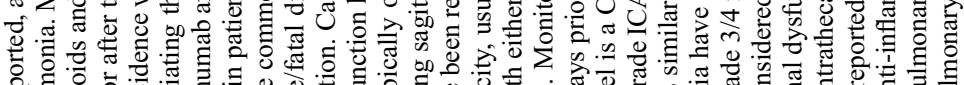

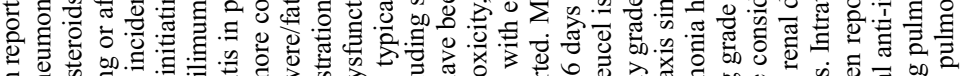

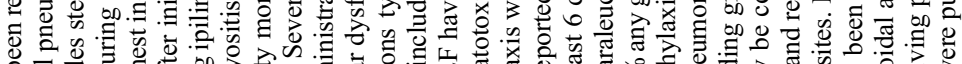
8.

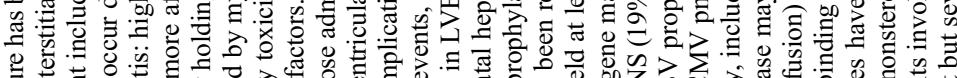

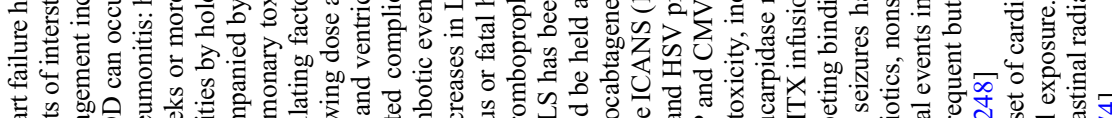

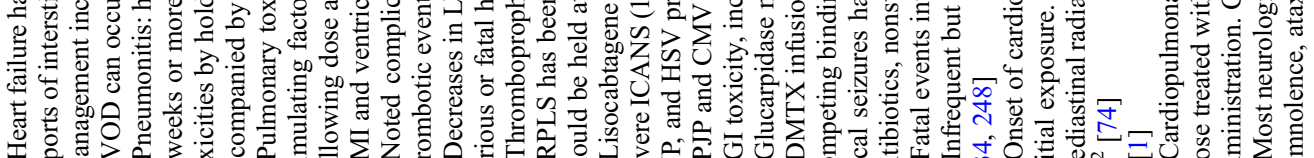

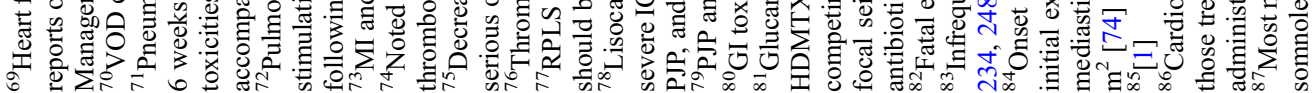


zั

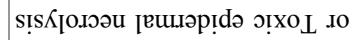

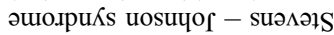
S!s К[оКшорqечу

气ै

్ㅗㅇ

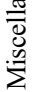

(วเәАәs) ภินเрәәด

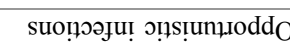

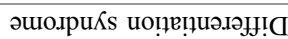

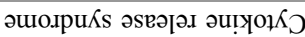

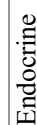

जิ

I

$\bar{৩}$

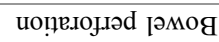

$\operatorname{s!̣!s} \kappa \mathrm{Ldod} \kappa_{\mathrm{H}}$

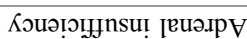

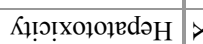

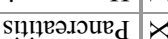

$\infty$ ஓ

z゙

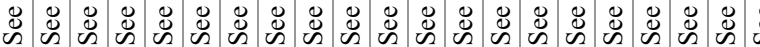

竎

范

苞

窇

를

ธ్융

ㅊํㅇ

क⿺尢丶

导氖

畨 过

穴

Dै

永

in

$=$ $m$ $m$ $\frac{\text { a }}{\frac{\pi}{E}}$ 를 : 药 $\frac{0}{0}$

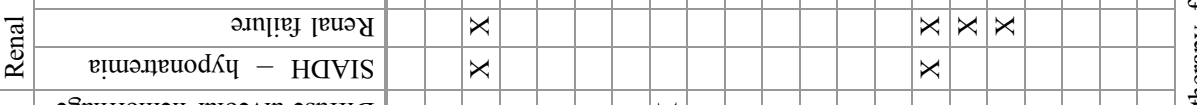

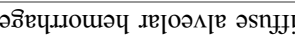

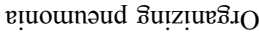

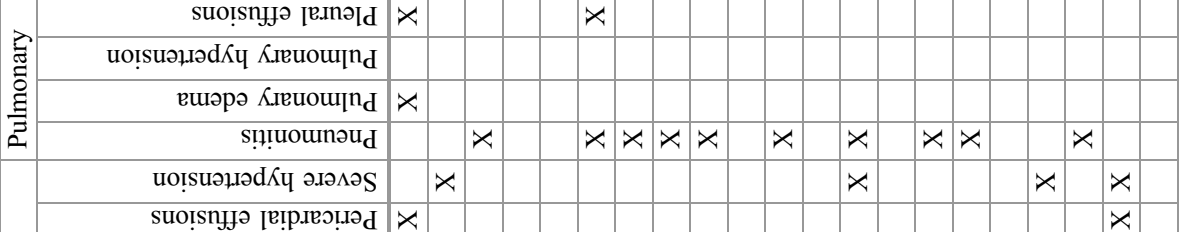

uọ̣อธินo!o.d IO

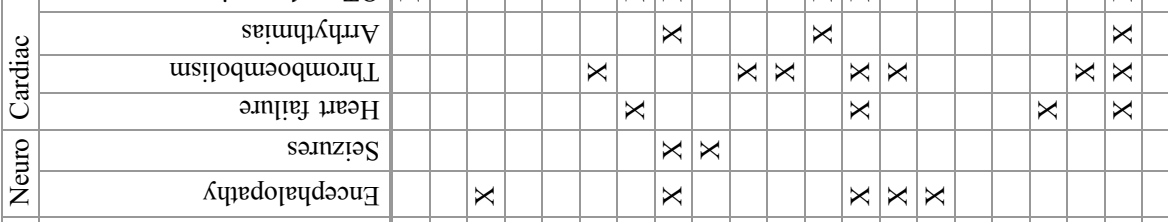




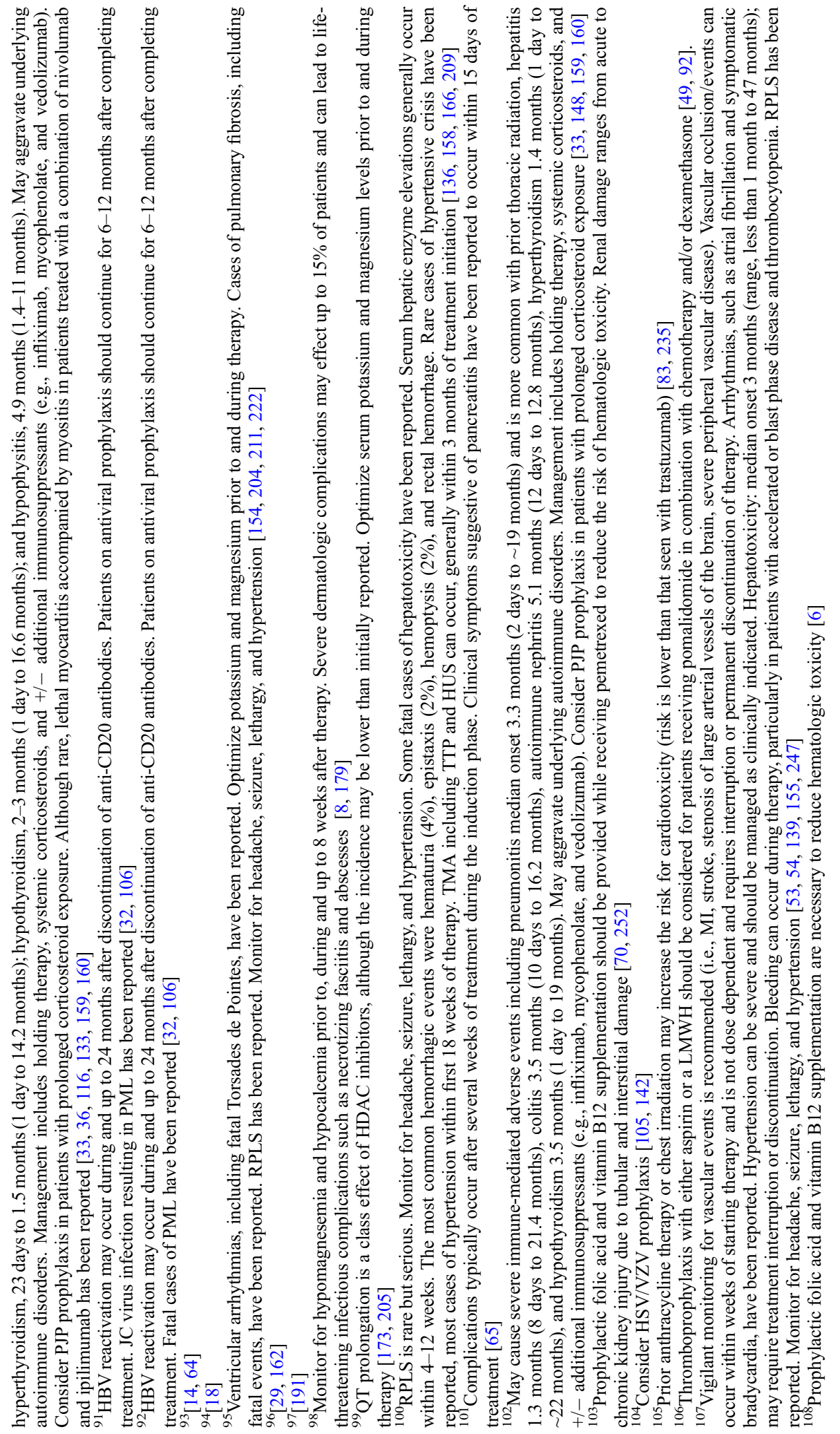




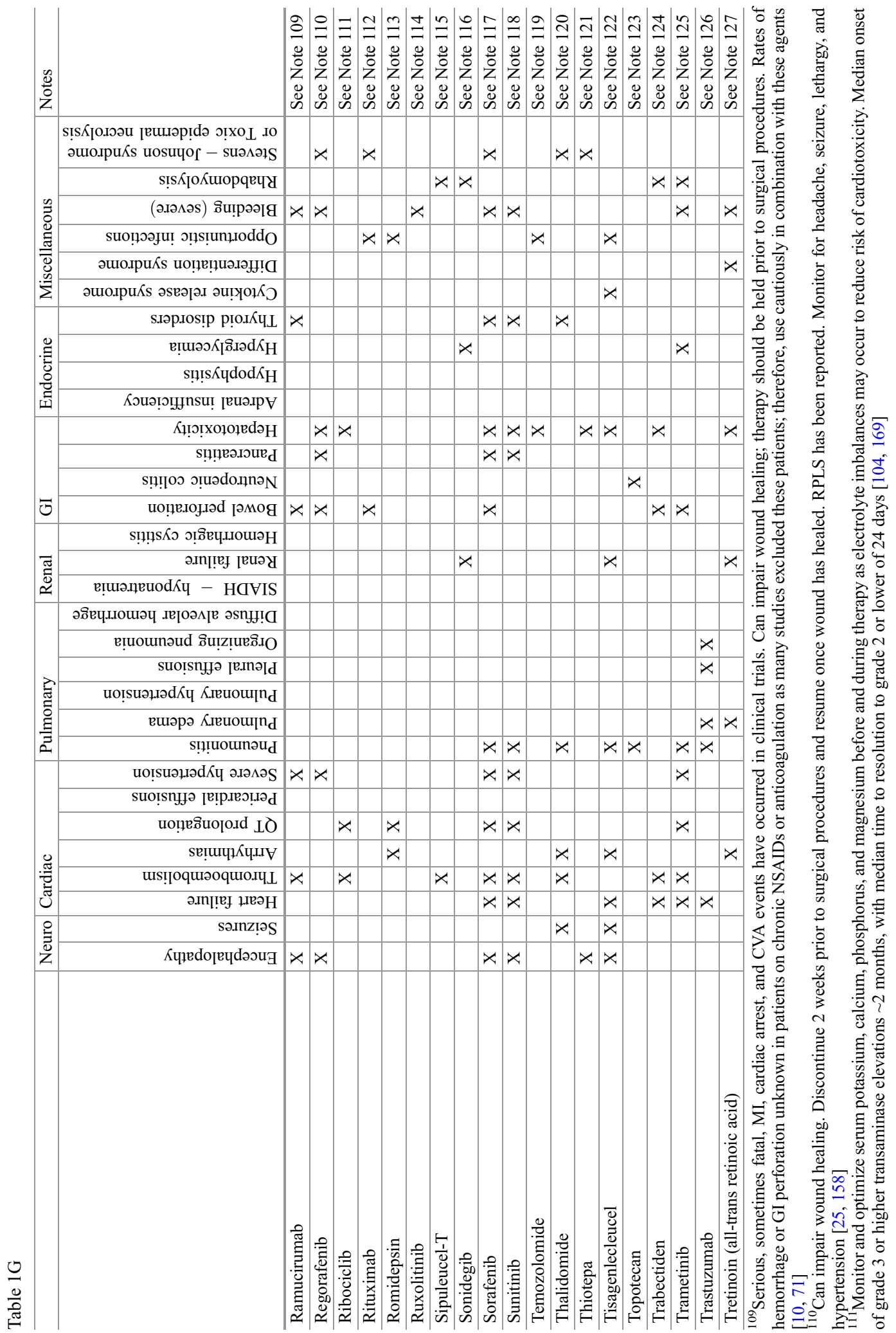




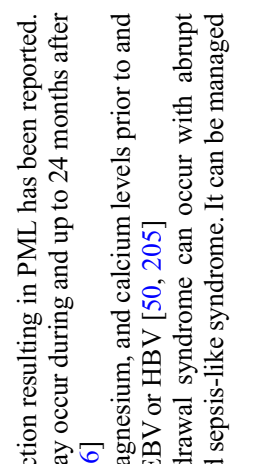

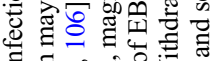

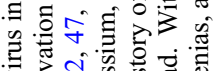

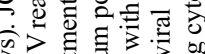

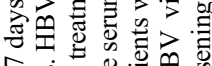

î.

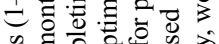

in

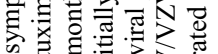

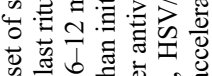

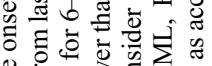

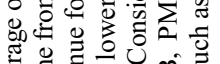

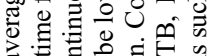

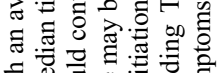

उ

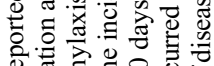

․ㅡㄹ

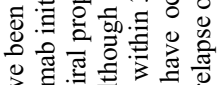

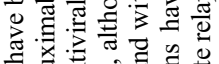

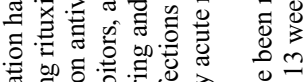

현

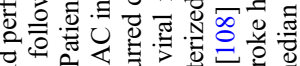

ธี

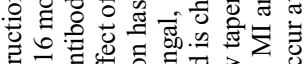

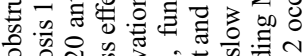

유유.

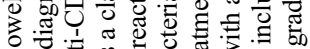

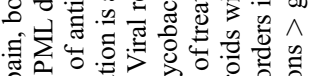

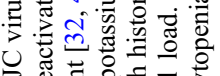

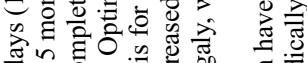

解产

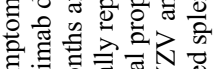

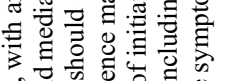

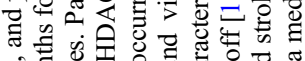

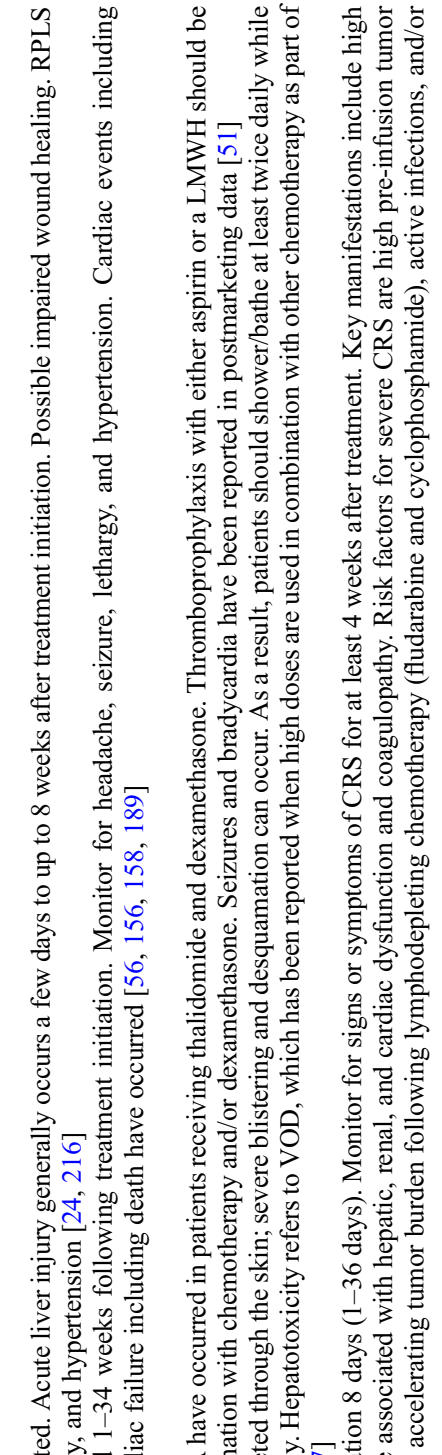

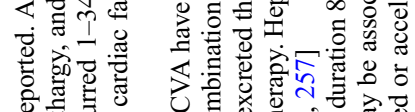

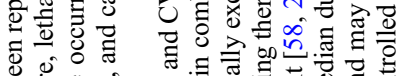

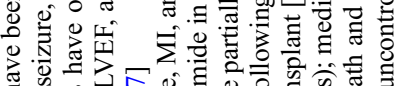

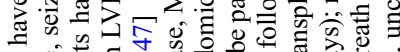
我

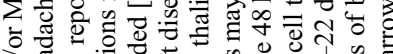

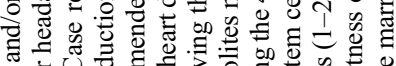

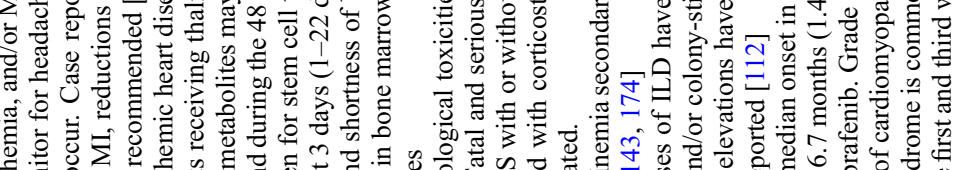

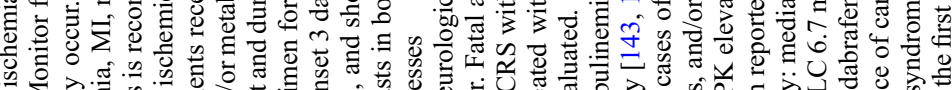

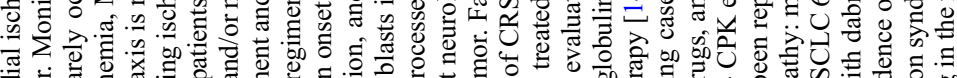

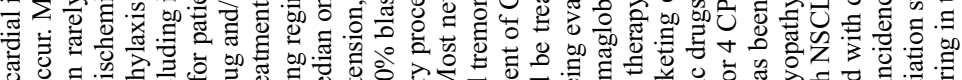

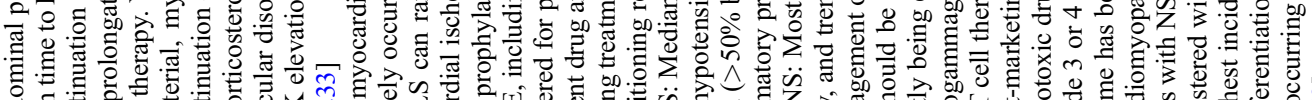

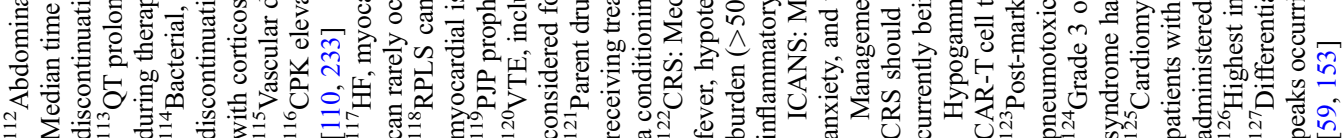




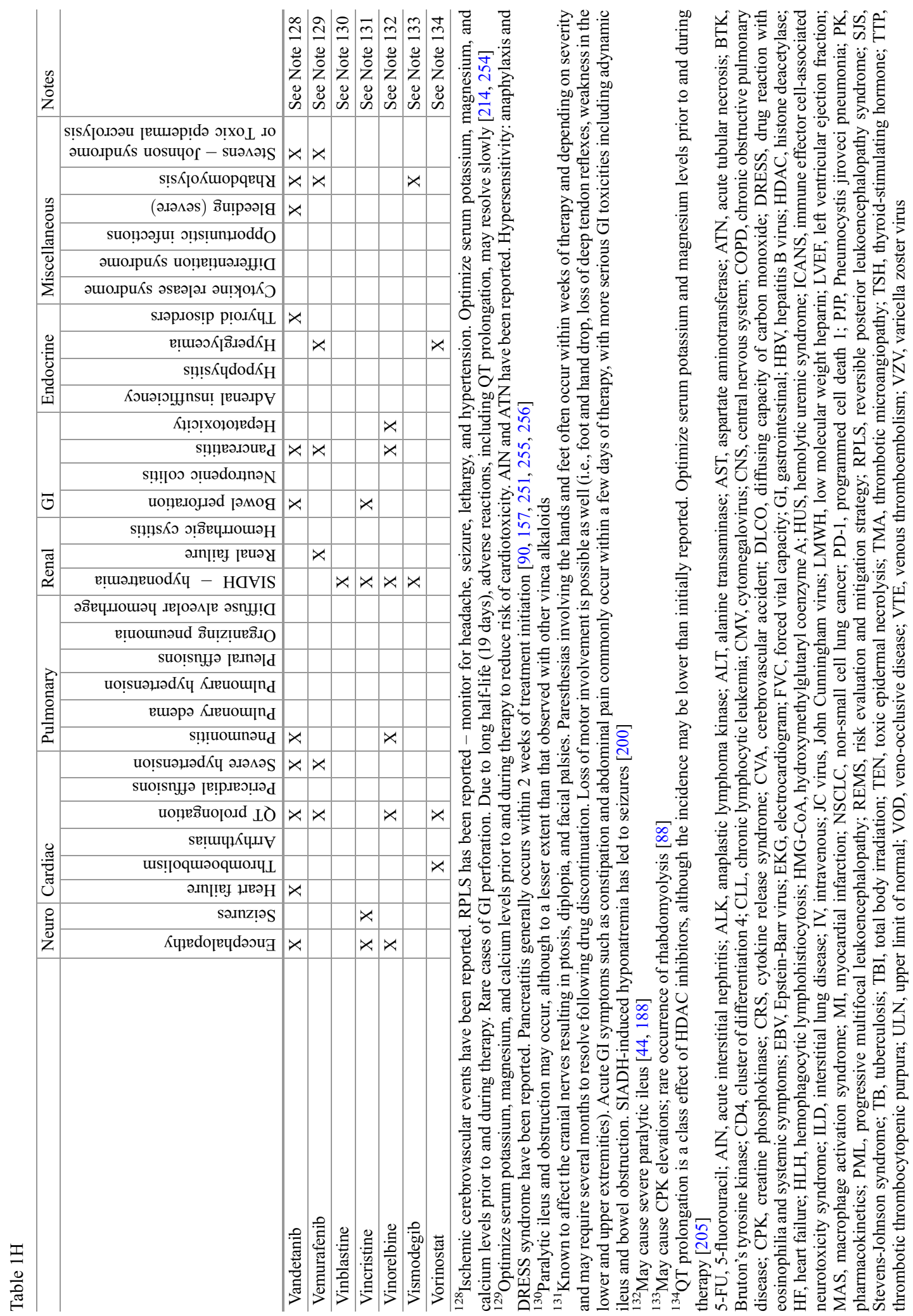




\section{References}

1. Abraxis Bioscience LLC. Abraxane (paclitaxel protein-bound particles) [package insert]. Summit; 2017.

2. Accord Healthcare Inc. Toposar (etoposide) [package insert]. Durham; 2014.

3. Ahn IE, Jerussi T, Farooqui M, Tian X, Wiestner A, Gea-Banacloche J. Atypical Pneumocystis jirovecii pneumonia in previously untreated patients with CLL on single-agent ibrutinib. Blood. 2016;128 (15):1940-3. https://doi.org/10.1182/blood-2016-06722991.

4. Aichberger KJ, Herndlhofer S, Schernthaner GH, Schillinger M, Mitterbauer-Hohendanner G, Sillaber C, Valent P. Progressive peripheral arterial occlusive disease and other vascular events during nilotinib therapy in CML. Am J Hematol. 2011;86 (7):533-9. https://doi.org/10.1002/ajh.22037.

5. Aldoss I, Douer D, Behrendt CE, Chaudhary P, Mohrbacher A, Vrona J, Pullarkat V. Toxicity profile of repeated doses of PEG-asparaginase incorporated into a pediatric-type regimen for adult acute lymphoblastic leukemia. Eur J Haematol. 2016;96 (4):375-80. https://doi.org/10.1111/ejh.12600.

6. Allos Therapeutics. Folotyn (pralatrexate) [package insert]. Westminister; 2009.

7. Amgen. Blincyto ${ }^{\circledR}$ (Blinatumomab) [package insert]. Thousand Oaks; 2017a.

8. Amgen. Vectibix (panitumumab) [package insert]. Thousand Oaks; 2017b.

9. Ariad Pharmaceuticals Inc. Alunbrig (brigatinib) [package insert]. Cambridge, MA; 2017.

10. Arnold D, Fuchs CS, Tabernero J, Ohtsu A, Zhu AX, Garon EB, Mackey JR, Paz-Ares L, Baron AD, Okusaka T, Yoshino T, Yoon HH, Das M, Ferry D, Zhang Y, Lin Y, Binder P, Sashegyi A, Chau I. Metaanalysis of individual patient safety data from six randomized, placebo-controlled trials with the antiangiogenic VEGFR2-binding monoclonal antibody ramucirumab. Ann Oncol. 2017;28(12):2932-42. https://doi.org/10.1093/annonc/mdx514.

11. Asbury RF, Rosenthal SN, Descalzi ME, Ratcliffe RL, Arseneau JC. Hepatic veno-occlusive disease due to DTIC. Cancer. 1980;45(10):2670-4.

12. AstraZeneca. Imfinzi (durvalumab) [package insert]. Wilmington; 2016.

13. AstraZeneca Pharmaceuticals. Calquence (acalabrutinib) [package insert]. Wilmington; 2017a.

14. AstraZeneca Pharmaceuticals. Lynparza (olaparib) capsules [package insert]. Wilmington; $2017 \mathrm{~b}$.

15. Au TH, Cavalieri CC, Stenehjem DD. Ceritinib: a primer for pharmacists. J Oncol Pharm Pract. 2017;23 (8):602-14. https://doi.org/10.1177/1078155216672315.

16. Azambuja E, Fleck JF, Batista RG, Menna Barreto SS. Bleomycin lung toxicity: who are the patients with increased risk? Pulm Pharmacol Ther. 2005;18 (5):363-6. https://doi.org/10.1016/j.pupt.2005.01.007.
17. AztraZeneca Pharmaceuticals LP. Iressa (gefitinib) [package insert]. Wilmington; 2015.

18. AztraZeneca Pharmaceuticals LP. Tagrisso (osimertinib) [package insert]. Wilmington; 2017.

19. Baker WJ, Royer GL Jr, Weiss RB. Cytarabine and neurologic toxicity. J Clin Oncol. 1991;9(4):679-93. https://doi.org/10.1200/jco.1991.9.4.679.

20. Baron M, Zini JM, Challan Belval T, Vignon M, Denis B, Alanio A, Malphettes M. Fungal infections in patients treated with ibrutinib: two unusual cases of invasive aspergillosis and cryptococcal meningoencephalitis. Leuk Lymphoma. 2017;58(12):2981-2. https://doi.org/10.1080/10428194.2017.1320710.

21. Baxter. Cyclophosphamide Injection [package insert]. Deerfield; 2015.

22. Baxter Healthcare Corp. Ifex (ifosfamide) [prescribing information]. Deerfield; 2014.

23. Bayer Healthcare Pharmaceuticals Inc. Aliqopa (copanlisib) [package insert]. Whippany; 2017a.

24. Bayer Healthcare Pharmaceuticals Inc. Nexevar (sorafenib) [package insert]. Whippany; 2017b.

25. Bayer HealthCare Pharmaceuticals Inc. Stivarga (regorafenib) [package insert]. Whippany; 2017c.

26. Bedford Laboratories. Adriamycin (doxorubicin HCL) [package insert]. Bedford; 2012.

27. Bedford Laboratories. Daunorubicin hydrochloride injection [package insert]. Bedford; 2013.

28. Bergeron A, Rea D, Levy V, Picard C, Meignin V, Tamburini J, Bruzzoni-Giovanelli H, Calvo F, Tazi A, Rousselot P. Lung abnormalities after dasatinib treatment for chronic myeloid leukemia: a case series. Am J Respir Crit Care Med. 2007;176(8):814-8. https:// doi.org/10.1164/rccm.200705-715CR.

29. Bielopolski D, Evron E, Moreh-Rahav O, Landes M, Stemmer SM, Salamon F. Paclitaxel-induced pneumonitis in patients with breast cancer: case series and review of the literature. J chemother (Florence, Italy). 2017;29(2):113-7. https://doi.org/10.1179/1973947 $815 y .0000000029$.

30. Blake-Haskins JA, Lechleider RJ, Kreitman RJ. Thrombotic microangiopathy with targeted cancer agents. Clin Cancer Res. 2011;17(18):5858-66. https://doi.org/10.1158/1078-0432.Ccr-11-0804.

31. Boehringer-ingelheim. Gilotrif (afatinib) [package insert]. Ridgfield; 2018.

32. Bohra C, Sokol L, Dalia S. Progressive multifocal leukoencephalopathy and monoclonal antibodies: a review. Cancer Control. 2017;24 (4):1073274817729901. https://doi.org/10.1177/107 3274817729901 .

33. Brahmer JR, Lacchetti C, Schneider BJ, Atkins MB, Brassil KJ, Caterino JM, Chau I, Ernstoff MS, Gardner JM, Ginex P, Hallmeyer S, Holter Chakrabarty J, Leighl NB, Mammen JS, McDermott DF, Naing A, Nastoupil LJ, Phillips T, Porter LD, Puzanov I, Reichner CA, Santomasso BD, Seigel C, Spira A, Suarez-Almazor ME, Wang Y, Weber JS, Wolchok JD, Thompson JA. Management of immune-related adverse events in patients treated with immune 
checkpoint inhibitor therapy: American society of clinical oncology clinical practice guideline. J Clin Oncol. 2018a;36:1714-68. https://doi.org/10.1200/ jco.2017.77.6385.

34. Brahmer JR, Lacchetti C, Schneider BJ, et al. Management of immune-related adverse events in patients treated with immune checkpoint inhibitor therapy: American society of clinical oncology clinical practice guideline. J Clin Oncol. 2018b;36:1714-68. (Published online: February 14, 2018).

35. Bristol-Myers Squibb. Ixempra (ixabepilone) [package insert]. Princeton; 2007.

36. Bristol-Myers Squibb. Opdivo (nivolumab) [package insert]. Princeton; 2018.

37. Bristol-Myers Squibb Company. Blenoxane (bleomycin) [package insert]. Princeton; 2010a.

38. Bristol-Myers Squibb Company. Platinol (cisplatin) [package insert]. Princeton; 2010b.

39. Bristol-Myers Squibb Company. Sprycel (dasatinib) [package insert]. Princeton; 2017a.

40. Bristol-Myers Squibb Company. Yervoy (ipilimumab) [package insert]. Princeton; 2017b.

41. Byrd JC, Harrington B, O'Brien S, Jones JA, Schuh A, Devereux S, Chaves J, Wierda WG, Awan FT, Brown JR, Hillmen P, Stephens DM, Ghia P, Barrientos JC, Pagel JM, Woyach J, Johnson D, Huang J, Wang X, Kaptein A, Lannutti BJ, Covey T, Fardis M, McGreivy J, Hamdy A, Rothbaum W, Izumi R, Diacovo TG, Johnson AJ, Furman RR. Acalabrutinib (ACP-196) in Relapsed Chronic Lymphocytic Leukemia. N Engl J Med. 2016;374(4):323-32. https://doi. org/10.1056/NEJMoa1509981.

42. Camacho LH. CTLA-4 blockade with ipilimumab: biology, safety, efficacy, and future considerations. Cancer Med. 2015;4(5):661-72. https://doi.org/ 10.1002/cam4.371.

43. Canellos GP, Anderson JR, Propert KJ, Nissen N, Cooper MR, Henderson ES, Green MR, Gottlieb A, Peterson BA. Chemotherapy of advanced Hodgkin's disease with MOPP, ABVD, or MOPP alternating with ABVD. N Engl J Med. 1992;327(21):1478-84. https://doi.org/10.1056/nejm199211193272102.

44. Canzler U, Schmidt-Gohrich UK, Bergmann S, Hanseroth K, Gatzweiler A, Distler W. Syndrome of inappropriate antidiuretic hormone secretion (SIADH) induced by vinorelbine treatment of metastatic breast cancer. Onkologie. 2007;30(8-9):455-6. https://doi.org/10.1159/000105143.

45. Carilli A, Favis G, Sundharkrishnan L, Hajdenberg J. Severe dermatologic reactions with bendamustine: a case series. Case Rep Oncol. 2014;7(2):465-70. https://doi.org/10.1159/000365324.

46. Carron PL, Cousin L, Caps T, Belle E, Pernet D, Neidhardt A, Capellier G. Gemcitabine-associated diffuse alveolar hemorrhage. Intensive Care Med. 2001;27 (9):1554. https://doi.org/10.1007/s001340101037.

47. Carson KR, Evens AM, Richey EA, Habermann TM, Focosi D, Seymour JF, Laubach J, Bawn SD, Gordon LI, Winter JN, Furman RR, Vose JM, Zelenetz AD,
Mamtani R, Raisch DW, Dorshimer GW, Rosen ST, Muro K, Gottardi-Littell NR, Talley RL, Sartor O, Green D, Major EO, Bennett CL. Progressive multifocal leukoencephalopathy after rituximab therapy in HIV-negative patients: a report of 57 cases from the Research on Adverse Drug Events and Reports project. Blood. 2009;113(20):4834-40. https://doi.org/ 10.1182/blood-2008-10-186999.

48. Carson KR, Newsome SD, Kim EJ, Wagner-Johnston ND, von Geldern G, Moskowitz CH, Moskowitz AJ, Rook AH, Jalan P, Loren AW, Landsburg D, Coyne T, Tsai D, Raisch DW, Norris LB, Bookstaver PB, Sartor O, Bennett CL. Progressive multifocal leukoencephalopathy associated with brentuximab vedotin therapy: a report of 5 cases from the Southern Network on Adverse Reactions (SONAR) project. Cancer. 2014;120(16):2464-71. https://doi.org/ 10.1002/cncr.28712.

49. Celegene Corporation. Pomalyst (pomalidomide) [package insert]. Summit; 2018.

50. Celgene Corporation. 2016 Istodax (romidepsin) [package insert]. Summit; 2018.

51. Celgene Corporation. Thalomid (thalidomide) [package insert]. Summit; 2017.

52. Ciurea SO, Andersson BS. Busulfan in hematopoietic stem cell transplantation. Biol Blood Marrow Transplant. 2009;15(5):523-36. https://doi.org/10.1016/j. bbmt.2008.12.489.

53. Cortes JE, Kantarjian H, Shah NP, Bixby D, Mauro MJ, Flinn I, O'Hare T, Hu S, Narasimhan NI, Rivera VM, Clackson T, Turner CD, Haluska FG, Druker BJ, Deininger MW, Talpaz M. Ponatinib in refractory Philadelphia chromosome-positive leukemias. N Engl J Med. 2012;367(22):2075-88. https://doi.org/ 10.1056/NEJMoa1205127.

54. Cortes JE, Kim DW, Pinilla-Ibarz J, le Coutre P, Paquette R, Chuah C, Nicolini FE, Apperley JF, Khoury HJ, Talpaz M, DiPersio J, DeAngelo DJ, Abruzzese E, Rea D, Baccarani M, Muller MC, Gambacorti-Passerini C, Wong S, Lustgarten S, Rivera VM, Clackson T, Turner CD, Haluska FG, Guilhot F, Deininger MW, Hochhaus A, Hughes T, Goldman JM, Shah NP, Kantarjian H. A phase 2 trial of ponatinib in Philadelphia chromosome-positive leukemias. N Engl J Med. 2013;369(19):1783-96. https://doi.org/10.1056/NEJMoa1306494.

55. Cortes JE, Saglio G, Kantarjian HM, Baccarani M, Mayer J, Boque C, Shah NP, Chuah C, Casanova L, BradleyGarelik B, Manos G, Hochhaus A. Final 5-year study results of DASISION: the dasatinib versus imatinib study in treatment-naive chronic myeloid leukemia patients trial. J Clin Oncol. 2016;34(20):2333-40. https://doi. org/10.1200/jco.2015.64.8899.

56. Costa R, Costa R, Costa R, Junior GM, Cartaxo HQ, de Barros AC. Reversible posterior encephalopathy syndrome secondary to sunitinib. Case Rep Oncol Med. 2014;2014:952624. https://doi.org/10.1155/ $2014 / 952624$. 
57. Cowman S, Stebbing J, Tuthill M. Large bowel perforation associated with capecitabine treatment for breast cancer. Ann Oncol. 2008;19(8):1510-1. https://doi.org/10.1093/annonc/mdn397.

58. Dalle JH, Giralt SA. Hepatic veno-occlusive disease after hematopoietic stem cell transplantation: risk factors and stratification, prophylaxis, and treatment. Biol Blood Marrow Transplant. 2016;22(3):400-9. https://doi.org/10.1016/j.bbmt.2015.09.024.

59. De Botton S, Dombret H, Sanz M, Miguel JS, Caillot D, Zittoun R, Gardembas M, Stamatoulas A, Conde E, Guerci A, Gardin C, Geiser K, Makhoul DC, Reman O, de la Serna J, Lefrere F, Chomienne C, Chastang C, Degos L, Fenaux P. Incidence, clinical features, and outcome of all trans-retinoic acid syndrome in 413 cases of newly diagnosed acute promyelocytic leukemia. The European APL Group. Blood. 1998;92(8):2712-8.

60. de Forni M, Malet-Martino MC, Jaillais P, Shubinski RE, Bachaud JM, Lemaire L, Canal P, Chevreau C, Carrie D, Soulie P, et al. Cardiotoxicity of high-dose continuous infusion fluorouracil: a prospective clinical study. J Clin Oncol. 1992;10(11):1795-801. https://doi.org/10.1200/jco.1992.10.11.1795.

61. Dendreon Corporation. Provenge (sipuleucel-T) [package insert]. Seattle; 2014.

62. Dhesi S, Chu MP, Blevins G, Paterson I, Larratt L, Oudit GY, Kim DH. Cyclophosphamide-induced cardiomyopathy: a case report, review, and recommendations for management. J Invest Med High Impact Case Rep. 2013;1(1):2324709613480346. https://doi. org/10.1177/2324709613480346.

63. Dimopoulos MA, Roussou M, Gavriatopoulou M, Psimenou E, Ziogas D, Eleutherakis-Papaiakovou E, Fotiou D, Migkou M, Kanellias N, Panagiotidis I, Ntalianis A, Papadopoulou E, Stamatelopoulos K, Manios E, Pamboukas C, Kontogiannis S, Terpos E, Kastritis E. Cardiac and renal complications of carfilzomib in patients with multiple myeloma. Blood Adv. 2017;1(7):449-54. https://doi.org/ 10.1182/bloodadvances.2016003269.

64. Domchek SM, Aghajanian C, Shapira-Frommer R, Schmutzler RK, Audeh MW, Friedlander M, Balmana J, Mitchell G, Fried G, Stemmer SM, Hubert A, Rosengarten O, Loman N, Robertson JD, Mann H, Kaufman B. Efficacy and safety of olaparib monotherapy in germline BRCA1/2 mutation carriers with advanced ovarian cancer and three or more lines of prior therapy. Gynecol Oncol. 2016;140(2):199-203. https://doi.org/10.1016/j.ygyno.2015.12.020.

65. Douer D, Aldoss I, Lunning MA, Burke PW, Ramezani L, Mark L, Vrona J, Park JH, Tallman MS, Avramis VI, Pullarkat V, Mohrbacher AM. Pharmacokinetics-based integration of multiple doses of intravenous pegaspargase in a pediatric regimen for adults with newly diagnosed acute lymphoblastic leukemia. J Clin Oncol. 2014;32(9):905-11. https://doi.org/10.1200/jco.2013.50.2708.
66. Dutcher JP, Schwartzentruber DJ, Kaufman HL, Agarwala SS, Tarhini AA, Lowder JN, Atkins MB. High dose interleukin-2 (Aldesleukin) - expert consensus on best management practices-2014. J Immuno Ther Cancer. 2014;2(1):26. https://doi.org/ 10.1186/s40425-014-0026-0.

67. Eisai Inc. Lenvima (lenvatinib) capsules [package insert]. Woodcliff Lake; 2017.

68. Eli Lilly and Company. Portrazza (necitumumab) [package insert]. Indianapolis; 2015.

69. Eli Lilly and Company. Erbitux (cetuximab) [package insert]. Indianapolis; 2016

70. Eli Lilly and Company. Alimta (pemetrexed) [package insert]. Indianapolis; 2017a.

71. Eli Lilly and Company. Cyramza (ramucirumab) [package insert]. Indianapolis; $2017 \mathrm{~b}$

72. Eli Lilly and Company. Gemzar (gemcitabine) [package insert]. Indianapolis; 2017c.

73. EMD Serono. Bavencio (avelumab) [package insert]. Rockland; 2017.

74. EMD Serono. Novantrone (Mitoxantrone) [package insert]. Rockland; 2008.

75. Eremina V, Jefferson JA, Kowalewska J, Hochster H, Haas M, Weisstuch J, Richardson C, Kopp JB, Kabir MG, Backx PH, Gerber HP, Ferrara N, Barisoni L, Alpers CE, Quaggin SE. VEGF inhibition and renal thrombotic microangiopathy. N Engl J Med. 2008;358(11):1129-36. https://doi.org/10.1056/NEJMoa0707330.

76. Exelixis Inc. Cometriq (cabozantinib) [package insert]. South San Francisco; 2017.

77. Faderl S, Ravandi F, Huang X, Garcia-Manero G, Ferrajoli A, Estrov Z, Borthakur G, Verstovsek S, Thomas DA, Kwari M, Kantarjian HM. A randomized study of clofarabine versus clofarabine plus low-dose cytarabine as front-line therapy for patients aged 60 years and older with acute myeloid leukemia and high-risk myelodysplastic syndrome. Blood. 2008;112 (5):1638-45. https://doi.org/10.1182/blood-2007-11124602.

78. Feinberg WM, Swenson MR. Cerebrovascular complications of L-asparaginase therapy. Neurology. 1988;38(1):127-33.

79. Gass-Jegu F, Gschwend A, Gairard-Dory AC, Mennecier B, Tebacher-Alt M, Gourieux B, Quoix E. Gastrointestinal perforations in patients treated with erlotinib: a report of two cases with fatal outcome and literature review. Lung Cancer (Amsterdam, Netherlands). 2016;99:76-8. https://doi.org/ 10.1016/j.lungcan.2016.06.012.

80. Geiser CF, Bishop Y, Jaffe N, Furman L, Traggis D, Frei E 3rd. Adverse effects of intrathecal methotrexate in children with acute leukemia in remission. Blood. 1975;45(2):189-95.

81. Genentech Inc. Herceptin (trastuzumab) [package insert]. South San Francisco; 2017a.

82. Genentech Inc. Kadcyla (ado-traztuzumab emtansine) [package insert]. South San Francisco; 2017b.

83. Genentech Inc. Perjeta (pertuzumab) [package insert]. South San Francisco; 2017c. 
84. Genentech Inc. Cotellic (cobimetinib) [package insert]. South San Francisco; 2018.

85. Genentech USA Inc. Xeloda (capecitabine) [package insert]. South San Francisco; 2016

86. Genentech USA Inc. Alecensa (alectinib) [package insert] South San Francisco; 2017a.

87. Genentech USA Inc. Avastin (bevacizumab) [package insert]. South San Francisco; 2017b.

88. Genentech USA Inc. Erivedge (vismodegib) [package insert]. South San Francisco; 2017c.

89. Genentech USA Inc. Tecentriq (atezolizumab) [package insert]. South San Francisco; 2017d.

90. Genentech USA Inc. Zelboraf (vemurafenib) [package insert]. South San Francisco; 2017e.

91. Genzyme Corporation. Lemtrada (alemtuzumab) [package insert]. Cambridge, MA; 2017.

92. Geyer HL, Viggiano RW, Lacy MQ, Witzig TE, Leslie KO, Mikhael JR, Stewart K. Acute lung toxicity related to pomalidomide. Chest. 2011;140 (2):529-33. https://doi.org/10.1378/chest.10-2082.

93. Gilead Sciences. Zydelig (idelalisib) [package insert]. Foster City; 2016.

94. Giles FJ, Mauro MJ, Hong F, Ortmann CE, McNeill C, Woodman RC, Hochhaus A, le Coutre PD, Saglio G. Rates of peripheral arterial occlusive disease in patients with chronic myeloid leukemia in the chronic phase treated with imatinib, nilotinib, or non-tyrosine kinase therapy: a retrospective cohort analysis. Leukemia. 2013;27(6):1310-5. https://doi. org/10.1038/leu.2013.69.

95. GlaxoSmithKline. Alkeran ${ }^{\circledR}$ (melphalan) [package insert]. Research Triangle Park; 2012.

96. Goldhar HA, Yan AT, Ko DT, Earle CC, Tomlinson GA, Trudeau ME, Krahn MD, Krzyzanowska MK, Pal RS, Brezden-Masley C, Gavura S, Lien K, Chan KK. The temporal risk of heart failure associated with adjuvant trastuzumab in breast cancer patients: a population study. J Natl Cancer Inst. 2016;108(1). https:// doi.org/10.1093/jnci/djv301.

97. Grellety T, Houede N, Hoepffner JL, Riviere J, Merino C, Lieutenant V, Gross-Goupil M, Richaud P, Dupin C, Sargos P, Roubaud G. Hemorrhagic cystitis in patients treated with cabazitaxel: a radiation recall syndrome? Ann Oncol. 2014;25(6):1248-9. https://doi.org/10.1093/ annonc/mdu 132 .

98. Hamnvik OP, Larsen PR, Marqusee E. Thyroid dysfunction from antineoplastic agents. J Natl Cancer Inst. 2011;103(21):1572-87. https://doi.org/10.1093/ jnci/djr373.

99. Haskell CM, Canellos GP, Leventhal BG, Carbone PP, Block JB, Serpick AA, Selawry OS. L-asparaginase: therapeutic and toxic effects in patients with neoplastic disease. N Engl J Med. 1969;281(19):1028-34. https:// doi.org/10.1056/nejm196911062811902.

100. Helman DL Jr, Byrd JC, Ales NC, Shorr AF. Fludarabine-related pulmonary toxicity: a distinct clinical entity in chronic lymphoproliferative syndromes. Chest. 2002;122(3):785-90.
101. Heritage Pharmaceuticals. BiCNU (carmustine) [package insert]. Eatontown; 2017.

102. Herzig RH, Hines JD, Herzig GP, Wolff SN, Cassileth PA, Lazarus HM, Adelstein DJ, Brown RA, Coccia PF, Strandjord S, et al. Cerebellar toxicity with high-dose cytosine arabinoside. J Clin Oncol. 1987;5(6):927-32. https://doi.org/10.1200/jco.1987.5.6.927.

103. Hochhaus A, Saglio G, Hughes TP, Larson RA, Kim DW, Issaragrisil S, le Coutre PD, Etienne G, DorlhiacLlacer PE, Clark RE, Flinn IW, Nakamae H, Donohue B, Deng W, Dalal D, Menssen HD, Kantarjian HM. Long-term benefits and risks of frontline nilotinib vs imatinib for chronic myeloid leukemia in chronic phase: 5-year update of the randomized ENESTnd trial. Leukemia. 2016;30(5):1044-54. https://doi.org/10.1038/leu.2016.5.

104. Hortobagyi GN, Stemmer SM, Burris HA, Yap YS, Sonke GS, Paluch-Shimon S, Campone M, Blackwell KL, Andre F, Winer EP, Janni W, Verma S, Conte P, Arteaga CL, Cameron DA, Petrakova K, Hart LL, Villanueva C, Chan A, Jakobsen E, Nusch A, Burdaeva O, Grischke EM, Alba E, Wist E, Marschner N, Favret AM, Yardley D, Bachelot T, Tseng LM, Blau S, Xuan F, Souami F, Miller M, Germa C, Hirawat S, O'Shaughnessy J. Ribociclib as first-line therapy for HR-positive, advanced breast cancer. N Engl J Med. 2016;375(18):1738-48. https://doi.org/10.1056/NEJMoa1609709.

105. Hospira. Nipent (pentostatin for injection) [package insert]. Lake Forest; 2018.

106. Hwang JP, Somerfield MR, Alston-Johnson DE, Cryer DR, Feld JJ, Kramer BS, Sabichi AL, Wong SL, Artz AS. Hepatitis B virus screening for patients with cancer before therapy: American society of clinical oncology provisional clinical opinion update. J Clin Oncol. 2015;33(19):2212-20. https://doi.org/ 10.1200/jco.2015.61.3745.

107. Ikeda S, Sekine A, Kato T, Yoshida M, Ogata R, Baba T, Nagahama K, Okudela K, Ogura T. Diffuse alveolar hemorrhage as a fatal adverse effect of bevacizumab: an autopsy case. Jpn J Clin Oncol. 2014;44(5):497-500. https://doi.org/10.1093/jjco/ hyu 023 .

108. Incyte Corp. Jakafi (ruxolitinib) [package insert]. Wilmington; 2011.

109. Ingenus Pharmaceuticals. Carboplatin [package insert]. Orlando; 2017.

110. Jain S, Song R, Xie J. Sonidegib: mechanism of action, pharmacology, and clinical utility for advanced basal cell carcinomas. Onco Targets Ther. 2017;10:1645-53. https://doi.org/10.2147/ott.S130910.

111. Janssen Biotech. Darzalex (daratumumab) [package insert]. Horsham; 2017.

112. Janssen Products. Yondelis (trabectedin) [package insert]. Horsham; 2015.

113. Janssen Products. Doxil (doxorubicin $\mathrm{HCl}$ liposome injection) [package insert]. Horsahm; 2016. 
114. Jazz Pharmaceuticals. VYXEOS (daunorubicin and cytarabine) liposome [package insert]. Palo Alto; 2018.

115. Jazz Pharmaceuticals. Erwinaze intramuscular injection, intravenous injection (asparaginase Erwinia chrysanthemi intramuscular injection, intravenous injection) [package insert]. Palo Alto; 2016.

116. Johnson DB, Balko JM, Compton ML, Chalkias S, Gorham J, Xu Y, Hicks M, Puzanov I, Alexander MR, Bloomer TL, Becker JR, Slosky DA, Phillips EJ, Pilkinton MA, Craig-Owens L, Kola N, Plautz G, Reshef DS, Deutsch JS, Deering RP, Olenchock BA, Lichtman AH, Roden DM, Seidman CE, Koralnik IJ, Seidman JG, Hoffman RD, Taube JM, Diaz LA Jr, Anders RA, Sosman JA, Moslehi JJ. Fulminant myocarditis with combination immune checkpoint blockade. N Engl J Med. 2016;375(18):1749-55. https:// doi.org/10.1056/NEJMoa1609214.

117. Johnston PB, Bondly C, Micallef IN. Ibritumomab tiuxetan for non-Hodgkin's lymphoma. Expert Rev Anticancer Ther. 2006;6(6):861-9. https://doi.org/ 10.1586/14737140.6.6.861.

118. Jolson HM, Bosco L, Bufton MG, Gerstman BB, Rinsler SS, Williams E, Flynn B, Simmons WD, Stadel BV, Faich GA, et al. Clustering of adverse drug events: analysis of risk factors for cerebellar toxicity with high-dose cytarabine. J Natl Cancer Inst. 1992;84(7):500-5.

119. June Therapeutics. Juno Therapeutics and Celgene corporation release additional data from TRANSCEND trial of JCAR017 in patients with relapsed or refractory aggressive B-cell non-Hodgkin lymphoma. 2017, viewed 11 March 2018. http://ir. celgene.com/releasedetail.cfm?releaseid $=1051336$

120. Junpaparp P, Sharma B, Samiappan A, Rhee JH, Young KR. Everolimus-induced severe pulmonary toxicity with diffuse alveolar hemorrhage. Ann Am Thorac Soc. 2013;10(6):727-9. https://doi.org/ 10.1513/AnnalsATS.201309-332LE.

121. Kadia TM, Gandhi V. Nelarabine in the treatment of pediatric and adult patients with T-cell acute lymphoblastic leukemia and lymphoma. Expert Rev Hematol. 2017;10(1):1-8. https://doi.org/10.1080/ 17474086.2017.1262757.

122. Kanie K, Iguchi G, Bando H, Fujita Y, Odake Y, Yoshida K, Matsumoto R, Fukuoka H, Ogawa W, Takahashi Y. Two cases of atezolizumab-induced hypophysitis. J Endocr Soc. 2018;2(1):91-5. https:// doi.org/10.1210/js.2017-00414.

123. Kantarjian HM, DeAngelo DJ, Advani AS, Stelljes M, Kebriaei P, Cassaday RD, Merchant AA, Fujishima N, Uchida T, Calbacho M, Ejduk AA, O'Brien SM, Jabbour EJ, Zhang H, Sleight BJ, Vandendries ER, Marks DI. Hepatic adverse event profile of inotuzumab ozogamicin in adult patients with relapsed or refractory acute lymphoblastic leukaemia: results from the open-label, randomised, phase 3 INO-VATE study. Lancet Haematol. 2017;4 (8):e387-98. https://doi.org/10.1016/s2352-3026(17) 30103-5.

124. Kebriaei P, Wilhelm K, Ravandi F, Brandt M, de Lima M, Ciurea S, Worth L, O'Brien S, Thomas D, Champlin RE, Kantarjian H. Feasibility of allografting in patients with advanced acute lymphoblastic leukemia after salvage therapy with inotuzumab ozogamicin. Clin Lymphoma Myeloma Leuk. 2013;13(3):296-301. https://doi.org/10.1016/j. clml.2012.12.003.

125. Khoury HJ, Cortes JE, Kantarjian HM, GambacortiPasserini C, Baccarani M, Kim DW, Zaritskey A, Countouriotis A, Besson N, Leip E, Kelly V, Brummendorf TH. Bosutinib is active in chronic phase chronic myeloid leukemia after imatinib and dasatinib and/or nilotinib therapy failure. Blood. 2012;119(15):3403-12. https://doi.org/10.1182/blood2011-11-390120.

126. Kieslich M, Porto L, Lanfermann H, Jacobi G, Schwabe D, Bohles H. Cerebrovascular complications of L-asparaginase in the therapy of acute lymphoblastic leukemia. J Pediatr Hematol Oncol. 2003;25(6):484-7.

127. Kim DW, Tiseo M, Ahn MJ, Reckamp KL, Hansen KH, Kim SW, Huber RM, West HL, Groen HJM, Hochmair MJ, Leighl NB, Gettinger SN, Langer CJ, Paz-Ares Rodriguez LG, Smit EF, Kim ES, Reichmann W, Haluska FG, Kerstein D, Camidge DR. Brigatinib in patients with crizotinib-refractory anaplastic lymphoma kinase-positive non-small-cell lung cancer: a randomized, multicenter phase II trial. J Clin Oncol. 2017;35(22):2490-8. https://doi.org/ 10.1200/jco.2016.71.5904.

128. Kite Pharma Inc. Yescarta (axicabtagene ciloleucel) [package insert]. Santa Monica; 2017.

129. Krause AS, Weihrauch MR, Bode U, Fleischhack G, Elter T, Heuer T, Engert A, Diehl V, Josting A. Carboxypeptidase-G2 rescue in cancer patients with delayed methotrexate elimination after high-dose methotrexate therapy. Leuk Lymphoma. 2002;43(11):2139-43. https://doi.org/10.1080/1042819021000032953.

130. Kuhlen M, Bleckmann K, Moricke A, Schrappe M, Vieth S, Escherich G, Bronsema A, Vonalt A, Queudeville M, Zwaan CM, Ebinger M, Debatin KM, Klingebiel T, Koscielniak E, Rossig C, Burkhardt B, Kolb R, Eckert C, Borkhardt A, von Stackelberg A, Chen-Santel C. Neurotoxic side effects in children with refractory or relapsed T-cell malignancies treated with nelarabine based therapy. Br J Haematol. 2017;179(2):272-83. https://doi.org/ 10.1111/bjh.14877.

131. Lambertini M, Del Mastro L, Gardin G, Levaggi A, Bighin C, Giraudi S, Pronzato P. Stevens-Johnson syndrome after treatment with bendamustine. Leuk Res. 2012;36(7):e153-4. https://doi.org/10.1016/j. leukres.2012.03.006.

132. Lancet JE, Uy G, Cortes JE, Newell LF, Lin TL, Ritchie EK, Stuart RK, Strickland SA, Hogge D, Solomon SR, Stone RM, Bixby DL, Kolitz JE, 
Schiller GJ, Wieduwilt MJ, Ryan DH, Hoering A, Chiarella M, Louie AC, Medeiros BC (Abstract 7000) Final results of a phase III randomized trial of CPX-351 versus $7+3$ in older patients with newly diagnosed high risk (secondary) AML. J Clin Oncol. 2016; 34(15 Suppl): p. 7000.

133. Larkin J, Chiarion-Sileni V, Gonzalez R, Grob JJ, Cowey CL, Lao CD, Schadendorf D, Dummer R, Smylie M, Rutkowski P, Ferrucci PF, Hill A, Wagstaff J, Carlino MS, Haanen JB, Maio M, Marquez-Rodas I, McArthur GA, Ascierto PA, Long GV, Callahan MK, Postow MA, Grossmann K, Sznol M, Dreno B, Bastholt L, Yang A, Rollin LM, Horak C, Hodi FS, Wolchok JD. Combined Nivolumab and ipilimumab or monotherapy in untreated melanoma. N Engl J Med. 2015;373 (1):23-34. https://doi.org/10.1056/NEJMoa1504030.

134. Latagliata $R$, Breccia $M$, Fava $C$, Stagno F, Tiribelli M, Luciano L, Gozzini A, Gugliotta G, Annunziata M, Cavazzini F, Ferrero D, Musto P, Capodanno I, Iurlo A, Visani G, Crugnola M, Calistri E, Castagnetti F, Vigneri P, Alimena G. Incidence, risk factors and management of pleural effusions during dasatinib treatment in unselected elderly patients with chronic myelogenous leukaemia. Hematol Oncol. 2013;31(2):103-9. https://doi.org/ 10.1002/hon.2020.

135. Le Coutre P, Rea D, Abruzzese E, Dombret H, Trawinska MM, Herndlhofer S, Dorken B, Valent P. Severe peripheral arterial disease during nilotinib therapy. J Natl Cancer Inst. 2011;103(17):1347-8. https://doi.org/10.1093/jnci/djr292.

136. Lenihan DJ, Kowey PR. Overview and management of cardiac adverse events associated with tyrosine kinase inhibitors. Oncologist. 2013;18(8):900-8. https://doi.org/10.1634/theoncologist.2012-0466.

137. Lilly USA LLC. Verzenio (abemaciclib) [package insert]. Indianapolis; 2017.

138. Linette DC, McGee KH, McFarland JA. Mitomycininduced pulmonary toxicity: case report and review of the literature. Ann Pharmacother. 1992;26(4):481-4. https://doi.org/10.1177/106002809202600404.

139. Lipton JH, Chuah C, Guerci-Bresler A, Rosti G, Simpson D, Assouline S, Etienne G, Nicolini FE, le Coutre P, Clark RE, Stenke L, Andorsky D, Oehler V, Lustgarten S, Rivera VM, Clackson T, Haluska FG, Baccarani M, Cortes JE, Guilhot F, Hochhaus A, Hughes T, Kantarjian HM, Shah NP, Talpaz M, Deininger MW. Ponatinib versus imatinib for newly diagnosed chronic myeloid leukaemia: an international, randomised, open-label, phase 3 trial. Lancet Oncol. 2016;17(5):612-21. https://doi.org/10.1016/ s1470-2045(16)00080-2.

140. Lyman GH, Bohlke K, Khorana AA, Kuderer NM, Lee AY, Arcelus JI, Balaban EP, Clarke JM, Flowers CR, Francis CW, Gates LE, Kakkar AK, Key NS, Levine MN, Liebman HA, Tempero MA, Wong SL, Somerfield MR, Falanga A. Venous thromboembolism prophylaxis and treatment in patients with cancer:
American society of clinical oncology clinical practice guideline update 2014. J Clin Oncol. 2015;33 (6):654-6. https://doi.org/10.1200/jco.2014.59.7351.

141. Malalagama GN, Chryssidis S, Parnis FX. CT findings in patients with Cabazitaxel induced pelvic pain and haematuria: a case series. Cancer Imaging. 2017;17(1):17. https://doi.org/10.1186/s40644-0170119-3.

142. Markman M, Kennedy A, Webster K, Elson P, Peterson G, Kulp B, Belinson J. Clinical features of hypersensitivity reactions to carboplatin. J Clin Oncol. 1999;17(4):1141. https://doi.org/10.1200/ jco.1999.17.4.1141.

143. Maude SL, Laetsch TW, Buechner J, Rives S, Boyer M, Bittencourt H, Bader P, Verneris MR, Stefanski HE, Myers GD, Qayed M, De Moerloose B, Hiramatsu H, Schlis K, Davis KL, Martin PL, Nemecek ER, Yanik GA, Peters C, Baruchel A, Boissel N, Mechinaud F, Balduzzi A, Krueger J, June CH, Levine BL, Wood P, Taran T, Leung M, Mueller KT, Zhang Y, Sen K, Lebwohl D, Pulsipher MA, Grupp SA. Tisagenlecleucel in children and young adults with B-Cell Lymphoblastic Leukemia. N Engl J Med. 2018;378(5):439-48. https://doi.org/10.1056/NEJMoa1709866.

144. Mayne Pharma Limited. Epirubicin hydrochloride [package insert]. Paramus; 2006.

145. McKoy JM, Angelotta C, Bennett CL, Tallman MS, Wadleigh M, Evens AM, Kuzel TM, Trifilio SM, Raisch DW, Kell J, DeAngelo DJ, Giles FJ. Gemtuzumab ozogamicin-associated sinusoidal obstructive syndrome (SOS): an overview from the research on adverse drug events and reports (RADAR) project. Leuk Res. 2007;31(5):599-604. https://doi.org/10.1016/j.leukres.2006.07.005.

146. McMullen JR, Boey EJ, Ooi JY, Seymour JF, Keating MJ, Tam CS. Ibrutinib increases the risk of atrial fibrillation, potentially through inhibition of cardiac PI3K-Akt signaling. Blood. 2014;124(25):3829-30. https://doi.org/10.1182/blood-2014-10-604272.

147. Merck \& Co. Temodar (temozolomide) [package insert]. Whitehouse Station; 2017a.

148. Merck \& Co. Keytruda (pembrolizumab) [package insert]. Whitehouse Station; $2017 \mathrm{~b}$.

149. Meyer CC, Calis KA, Burke LB, Walawander CA, Grasela TH. Symptomatic cardiotoxicity associated with 5-fluorouracil. Pharmacotherapy. 1997;17 (4):729-36.

150. Millennium Pharmaceuticals Inc. Velcade (bortezomib) [package insert]. Cambridge, MA; 2017.

151. Mitani S, Kadowaki S, Komori A, Sugiyama K, Narita Y, Taniguchi H, Ura T, Ando M, Sato Y, Yamaura H, Inaba Y, Ishihara M, Tanaka T, Tajika M, Muro K. Acute hyperammonemic encephalopathy after fluoropyrimidine-based chemotherapy: a case series and review of the literature. Medicine. 2017;96(22):e6874. https://doi.org/10.1097/md.0000000000006874. 
152. Montani D, Bergot E, Gunther S, Savale L, Bergeron A, Bourdin A, Bouvaist $\mathrm{H}$, Canuet M, Pison C, Macro M, Poubeau P, Girerd B, Natali D, Guignabert C, Perros F, O'Callaghan DS, Jais X, Tubert-Bitter P, Zalcman G, Sitbon O, Simonneau G, Humbert M. Pulmonary arterial hypertension in patients treated by dasatinib. Circulation. 2012;125(17):2128-37. https://doi.org/10.1161/ circulationaha.111.079921.

153. Montesinos P, Bergua JM, Vellenga E, Rayon C, Parody R, de la Serna J, Leon A, Esteve J, Milone G, Deben G, Rivas C, Gonzalez M, Tormo M, Diaz-Mediavilla J, Gonzalez JD, Negri S, Amutio E, Brunet S, Lowenberg B, Sanz MA. Differentiation syndrome in patients with acute promyelocytic leukemia treated with all-trans retinoic acid and anthracycline chemotherapy: characteristics, outcome, and prognostic factors. Blood. 2009;113 (4):775-83. https://doi.org/10.1182/blood-2008-07168617.

154. Moskovitz M, Wollner M, Haim N. Oxaliplatininduced pulmonary toxicity in gastrointestinal malignancies: two case reports and review of the literature. Case Rep Oncol Med. 2015;2015:341064. https://doi. org/10.1155/2015/341064.

155. Moslehi JJ, Deininger M. Tyrosine Kinase InhibitorAssociated Cardiovascular Toxicity in Chronic Myeloid Leukemia. J Clin Oncol. 2015;33(35):4210-8. https://doi.org/10.1200/jco.2015.62.4718.

156. Motzer RJ, Hutson TE, Olsen MR, Hudes GR, Burke JM, Edenfield WJ, Wilding G, Agarwal N, Thompson JA, Cella D, Bello A, Korytowsky B, Yuan J, Valota O, Martell B, Hariharan S, Figlin RA. Randomized phase II trial of sunitinib on an intermittent versus continuous dosing schedule as first-line therapy for advanced renal cell carcinoma. J Clin Oncol. 2012;30(12):1371-7. https://doi.org/ 10.1200/jco.2011.36.4133.

157. Munch M, Peuvrel L, Brocard A, Saint Jean M, Khammari A, Dreno B, Quereux G. Early-onset vemurafenib-induced DRESS syndrome. Dermatology (Basel, Switzerland). 2016;232(1):126-8. https://doi.org/10.1159/000439272.

158. Myint ZW, Sen JM, Watts NL, Druzgal TJ, Nathan BR, Ward MD, Boyer JE, Fracasso PM. Reversible posterior leukoencephalopathy syndrome during regorafenib treatment: a case report and literature review of reversible posterior leukoencephalopathy syndrome associated with multikinase inhibitors. Clin Colorectal Cancer. 2014;13(2):127-30. https:// doi.org/10.1016/j.clcc.2013.12.003.

159. Naidoo J, Page DB, Li BT, Connell LC, Schindler K, Lacouture ME, Postow MA, Wolchok JD. Toxicities of the anti-PD-1 and anti-PD-L1 immune checkpoint antibodies. Ann Oncol. 2015;26(12):2375-91. https://doi.org/10.1093/annonc/mdv383.

160. Naidoo J, Wang X, Woo KM, Iyriboz T, Halpenny D, Cunningham J, Chaft JE, Segal NH, Callahan MK, Lesokhin AM, Rosenberg J, Voss MH, Rudin CM,
Rizvi H, Hou X, Rodriguez K, Albano M, Gordon RA, Leduc C, Rekhtman N, Harris B, Menzies AM, Guminski AD, Carlino MS, Kong BY, Wolchok JD, Postow MA, Long GV, Hellmann MD. Pneumonitis in patients treated with anti-programmed death-1/programmed death ligand 1 therapy. J Clin Oncol. 2017;35 (7):709-17. https://doi.org/10.1200/jco.2016.68.2005.

161. Neelapu SS, Locke FL, Bartlett NL, Lekakis LJ, Miklos DB, Jacobson CA, Braunschweig I, Oluwole OO, Siddiqi T, Lin Y, Timmerman JM, Stiff PJ, Friedberg JW, Flinn IW, Goy A, Hill BT, Smith MR, Deol A, Farooq U, McSweeney P, Munoz J, Avivi I, Castro JE, Westin JR, Chavez JC, Ghobadi A, Komanduri KV, Levy R, Jacobsen ED, Witzig TE, Reagan P, Bot A, Rossi J, Navale L, Jiang Y, Aycock J, Elias M, Chang D, Wiezorek J, Go WY. Axicabtagene ciloleucel CAR T-Cell therapy in refractory large B-Cell lymphoma. N Engl J Med. 2017;377(26):2531-44. https://doi.org/10.1056/ NEJMoa1707447.

162. Nesher L, Rolston KV. Neutropenic enterocolitis, a growing concern in the era of widespread use of aggressive chemotherapy. Clin Infect Dis. 2013;56 (5):711-7. https://doi.org/10.1093/cid/cis998.

163. NIH NCI. A to Z list of cancer drugs; 2018. Viewed 20 March 2018. https://www.cancer.gov/about-can cer/treatment/drugs

164. Novartis Pharmaceutical Co. Afinitor and Afinitor Disperz (everolimus) [package insert]. East Hanover; 2017a.

165. Novartis Pharmaceutical Co. Tykerb (Lapatinib) [package insert]. East Hanover; $2017 \mathrm{~b}$.

166. Novartis Pharmaceutical Co. Votrient (pazopanib) [package insert]. East Hanover; 2017c.

167. Novartis Pharmaceutical Co. Zykadia (ceritinib) [package insert] East Hanover; 2017d.

168. Novartis Pharmaceuticals. Hycamtin (topotecan oral capsules) [package insert]. East Hanover; 2016.

169. Novartis Pharmaceuticals. Kasqali (ribociclib) [package insert]. East Hanover; 2017a.

170. Novartis Pharmaceuticals. Mekinist (trametinib) [package insert]. East Hanover; $2017 \mathrm{~b}$.

171. Novartis Pharmaceuticals. Tafinlar (dabrafenib) [package insert]. East Hanover; 2017c.

172. Novartis Pharmaceuticals. Tasigna oral capsules, nilotinib oral capsules [package insert]. East Hanover; 2018.

173. Novartis Pharmaceuticals Corporation. Farydak (panobinostat) [package insert]. East Hanover; 2016.

174. Novartis Pharmaceuticals Corporation. Kymriah (tisagenlecleucel) [prescribing information]. East Hanover; 2017.

175. Ohnishi K, Sakai F, Kudoh S, Ohno R. Twenty-seven cases of drug-induced interstitial lung disease associated with imatinib mesylate. Leukemia. 2006;20 (6):1162-4. https://doi.org/10.1038/sj.leu.2404207.

176. Okamoto K, Proia LA, Demarais PL. Disseminated cryptococcal disease in a patient with chronic lymphocytic leukemia on ibrutinib. Case Rep Infect Dis. 
2016;2016:4642831. https://doi.org/10.1155/2016/ 4642831.

177. Okuno SH, Frytak S. Mitomycin lung toxicity. Acute and chronic phases. Am J Clin Oncol. 1997;20 (3):282-4.

178. Onyx Pharmaceuticals Inc. Kyprolis (carfilzomib) [package insert]. Thousand Oaks; 2018.

179. Osawa M, Kudoh S, Sakai F, Endo M, Hamaguchi T, Ogino Y, Yoneoka M, Sakaguchi M, Nishimoto H, Gemma A. Clinical features and risk factors of panitumumab-induced interstitial lung disease: a postmarketing all-case surveillance study. Int J Clin Oncol. 2015;20(6):1063-71. https://doi.org/10.1007/ s10147-015-0834-3.

180. OSI Pharmaceuticals L, an affiliate of Astellas Pharma US, Inc. Tarceva (erlotinib) [package insert]. Northbrook; 2016.

181. Otsuka America Pharmaceutical. Busulfan [package insert]. Rockville; 2015.

182. Ovation Pharmaceuticals. Cosmegen (dactinomycin) [package insert]. Deerfield; 2008.

183. Patel PN. Methylene blue for management of Ifosfamide-induced encephalopathy. Ann Pharmacother. 2006;40(2):299-303. https://doi.org/ 10.1345/aph.1G114.

184. Perez EA, Koehler M, Byrne J, Preston AJ, Rappold E, Ewer MS. Cardiac safety of lapatinib: pooled analysis of 3689 patients enrolled in clinical trials. Mayo Clin Proc. 2008;83(6):679-86. https:// doi.org/10.4065/83.6.679.

185. Peroukides S, Makatsoris T, Koutras A, Tsamandas A, Onyenadum A, Labropoulou-KaratzaC, Kalofonos H. Lapatinib-induced hepatitis: a case report. World J Gastroenterol. 2011;17(18):2349-52. https://doi.org/10.3748/wjg.v17.i18.2349.

186. Petri CR, O'Donnell PH, Cao H, Artz AS, Stock W, Wickrema A, Hard M, van Besien K. Clofarabineassociated acute kidney injury in patients undergoing hematopoietic stem cell transplant. Leuk Lymphoma. 2014;55(12):2866-73. https://doi.org/10.3109/1042 8194.2014.897701.

187. Pfizer. Inlyta (axitinib) [package insert]. New York; 2014.

188. Pfizer. Navelbine (vinorelbine) [package insert]; Research Triangle Park; 2017a.

189. Pfizer. Sutent (sunitinib) [package insert]. New York; 2017b.

190. Pfizer Inc. Xalkori (crizotinib) [package insert]. New York; 2017.

191. Pfizer Labs. Ibrance (palbociclib) [package insert]. New York; 2017.

192. Pharmacia \& Upjohn Camptosar (irinotecan) [package insert]. New York; 2016.

193. Pharmacyclics. Imbruvica (ibrutinib) [package insert]. Sunnyvale; 2017.

194. Porkka K, Khoury HJ, Paquette RL, Matloub Y, Sinha R, Cortes JE. Dasatinib $100 \mathrm{mg}$ once daily minimizes the occurrence of pleural effusion in patients with chronic myeloid leukemia in chronic phase and efficacy is unaffected in patients who develop pleural effusion. Cancer. 2010;116 (2):377-86. https://doi.org/10.1002/cncr.24734.

195. Prasco Laboratories. Leukeran (chlorambucil) [package insert]. Mason; 2017.

196. Prometheus Laboratories Inc. Proleukin (aldesleukin) [prescribing information]. San Diego; 2012.

197. Qi WX, Fu S, Zhang Q, Guo XM. Bevacizumab increases the risk of severe congestive heart failure in cancer patients: an up-to-date meta-analysis with a focus on different subgroups. Clin Drug Investig. 2014;34(10):681-90. https://doi.org/10.1007/s40261014-0222-1.

198. Quintas-Cardama A, Kantarjian H, Cortes J. Nilotinib-associated vascular events. Clin Lymphoma Myeloma Leuk. 2012;12(5):337-40. https:// doi.org/10.1016/j.clml.2012.04.005.

199. Read WL, Mortimer JE, Picus J. Severe interstitial pneumonitis associated with docetaxel administration. Cancer. 2002;94(3):847-53.

200. Robertson GL, Bhoopalam N, Zelkowitz LJ. Vincristine neurotoxicity and abnormal secretion of antidiuretic hormone. Arch Intern Med. 1973;132 (5):717-20.

201. Roboz GJ, Ritchie EK, Carlin RF, Samuel M, Gale L, Provenzano-Gober JL, Curcio TJ, Feldman EJ, Kligfield PD. Prevalence, management, and clinical consequences of QT interval prolongation during treatment with arsenic trioxide. J Clin Oncol. 2014;32 (33):3723-8. https://doi.org/10.1200/jco.2013.51.2913.

202. Rosen AC, Balagula Y, Raisch DW, Garg V, Nardone B, Larsen N, Sorrell J, West DP, Anadkat MJ, Lacouture ME. Life-threatening dermatologic adverse events in oncology. Anti-Cancer Drugs. 2014;25(2):225-34. https://doi.org/10.1097/cad.0000000000000032.

203. Rothenstein JM, Letarte N. Managing treatmentrelated adverse events associated with Alk inhibitors. Curr Oncol (Toronto, Ont). 2014;21(1):19-26. https://doi.org/10.3747/co.21.1740.

204. Rubbia-Brandt L, Audard V, Sartoretti P, Roth AD, Brezault C, Le Charpentier M, Dousset B, Morel P, Soubrane O, Chaussade S, Mentha G, Terris B. Severe hepatic sinusoidal obstruction associated with oxaliplatin-based chemotherapy in patients with metastatic colorectal cancer. Ann Oncol. 2004;15(3):460-6.

205. Sager PT, Balser B, Wolfson J, Nichols J, Pilot R, Jones S, Burris HA. Electrocardiographic effects of class 1 selective histone deacetylase inhibitor romidepsin. Cancer Med. 2015;4(8):1178-85. https://doi.org/10.1002/cam4.467.

206. Saif MW, McGee PJ. Hemolytic-uremic syndrome associated with gemcitabine: a case report and review of literature. J Pancreas. 2005;6(4):369-74.

207. Saif MW, Tomita M, Ledbetter L, Diasio RB. Capecitabine-related cardiotoxicity: recognition and management. J Support Oncol. 2008;6(1):41-8.

208. Salloum E, Khan KK, Cooper DL. Chlorambucilinduced seizures. Cancer. 1997;79(5):1009-13. 
209. Sankhalla KK, Chawla NS, Syed I, Chawla SP. Management of toxicities with pazopanib in advanced soft tissue sarcoma. Sarcoma Res Int. 2016;3(2):1032-8.

210. Sanofi-Aventis. Zaltrapt (afilibercept) [package insert]. Bridgewater; 2016.

211. Sanofi-Aventis LLC. Eloxatin (oxaliplatin) [package insert]. Bridgewater; 2015.

212. Sanofi-Aventis U.S. LLC. Taxotere (docetaxel) [package insert]. Bridgewater; 2015.

213. Sanofi-Aventis U.S. LLC. Jevtana (cabazitaxel) [package insert]. Bridgewater; 2017.

214. Sanofi Genzyme Corp. Caprelsa (vandetanib) [package insert]. Cambridge, MA; 2016.

215. Sanz MA, Montesinos P. How we prevent and treat differentiation syndrome in patients with acute promyelocytic leukemia. Blood. 2014;123(18):2777-82. https://doi.org/10.1182/blood-2013-10-512640.

216. Schmidinger M, Zielinski CC, Vogl UM, Bojic A, Bojic M, Schukro C, Ruhsam M, Hejna M, Schmidinger H. Cardiac toxicity of sunitinib and sorafenib in patients with metastatic renal cell carcinoma. J Clin Oncol. 2008;26(32):5204-12. https:// doi.org/10.1200/jco.2007.15.6331.

217. Seattle Genetics. Adcetris (brentuximab vedotin) [package insert]. Bothell; 2017.

218. Seet RC, Rabinstein AA. Clinical features and outcomes of posterior reversible encephalopathy syndrome following bevacizumab treatment. QJM. 2012;105(1):69-75. https://doi.org/10.1093/qjmed/ her139.

219. Serota DP, Mehta AK, Phadke VK. Invasive fungal sinusitis due to Mucor species in a patient on ibrutinib. Clin Infect Dis. 2017; https://doi.org/ 10.1093/cid/cix1058.

220. Shah NP, Kantarjian HM, Kim DW, Rea D, DorlhiacLlacer PE, Milone JH, Vela-Ojeda J, Silver RT, Khoury HJ, Charbonnier A, Khoroshko N, Paquette RL, Deininger M, Collins RH, Otero I, Hughes T, Bleickardt E, Strauss L, Francis S, Hochhaus A. Intermittent target inhibition with dasatinib $100 \mathrm{mg}$ once daily preserves efficacy and improves tolerability in imatinib-resistant and -intolerant chronic-phase chronic myeloid leukemia. J Clin Oncol. 2008;26(19):3204-12. https://doi.org/ 10.1200/jco.2007.14.9260.

221. Shank BR, Do B, Sevin A, Chen SE, Neelapu SS, Horowitz SB. Chimeric Antigen Receptor T Cells in Hematologic Malignancies. Pharmacotherapy. 2017;37 (3):334 45. https://doi.org/10.1002/phar.1900.

222. Sharief U, Perry DJ. Delayed reversible posterior encephalopathy syndrome following chemotherapy with oxaliplatin. Clin Colorectal Cancer. 2009;8 (3):163-5. https://doi.org/10.3816/CCC.2009.n.026.

223. Shaw AT, Kim DW, Nakagawa K, Seto T, Crino L, Ahn MJ, De Pas T, Besse B, Solomon BJ, Blackhall F, Wu YL, Thomas M, O'Byrne KJ, Moro-Sibilot D, Camidge DR, Mok T, Hirsh V, Riely GJ, Iyer S, Tassell V, Polli A, Wilner KD, Janne PA. Crizotinib versus chemotherapy in advanced ALK-positive lung cancer. N Engl J Med. 2013;368(25):2385-94. https://doi.org/10.1056/NEJMoa1214886.

224. Sievers EL, Larson RA, Stadtmauer EA, Estey E, Lowenberg B, Dombret H, Karanes C, Theobald M, Bennett JM, Sherman ML, Berger MS, Eten CB, Loken MR, van Dongen JJ, Bernstein ID, Appelbaum FR. Efficacy and safety of gemtuzumab ozogamicin in patients with CD33-positive acute myeloid leukemia in first relapse. J Clin Oncol. 2001;19(13):3244-54. https://doi.org/10.1200/jco.2001.19.13.3244.

225. Sledge GW Jr, Toi M, Neven P, Sohn J, Inoue K, Pivot X, Burdaeva O, Okera M, Masuda N, Kaufman PA, Koh H, Grischke EM, Frenzel M, Lin Y, Barriga S, Smith IC, Bourayou N, Llombart-Cussac A. MONARCH 2: abemaciclib in combination with fulvestrant in women with HR+/HER2-advanced breast cancer who had progressed while receiving endocrine therapy. J Clin Oncol. 2017;35(25):2875-84. https://doi. org/10.1200/jco.2017.73.7585.

226. Smith GA, Damon LE, Rugo HS, Ries CA, Linker CA. High-dose cytarabine dose modification reduces the incidence of neurotoxicity in patients with renal insufficiency. J Clin Oncol. 1997;15(2):833-9. https://doi.org/10.1200/jco.1997.15.2.833.

227. Soria JC, Tan DSW, Chiari R, Wu YL, Paz-Ares L, Wolf J, Geater SL, Orlov S, Cortinovis D, Yu CJ, Hochmair M, Cortot AB, Tsai CM, Moro-Sibilot D, Campelo RG, McCulloch T, Sen P, Dugan M, Pantano S, Branle F, Massacesi C, de Castro G Jr. First-line ceritinib versus platinum-based chemotherapy in advanced ALK-rearranged non-small-cell lung cancer (ASCEND-4): a randomised, open-label, phase 3 study. Lancet (London, England). 2017;389 (10072):917-29. https://doi.org/10.1016/s0140-6736 (17)30123-x.

228. Spectrum Pharmaceuticals Beleodaq (belinostat) [package insert]. Irvine; 2017.

229. Steeghs N, de Jongh FE, Sillevis Smitt PA, van den Bent MJ. Cisplatin-induced encephalopathy and seizures. Anti-Cancer Drugs. 2003;14(6):443-6. https:// doi.org/10.1097/01.cad.0000078733.65608.38.

230. Stein EM, DiNardo CD, Pollyea DA, Fathi AT, Roboz GJ, Altman JK, Stone RM, DeAngelo DJ, Levine RL, Flinn IW, Kantarjian HM, Collins R, Patel MR, Frankel AE, Stein A, Sekeres MA, Swords RT, Medeiros BC, Willekens C, Vyas P, Tosolini A, $\mathrm{Xu} \mathrm{Q}$, Knight RD, Yen KE, Agresta S, de Botton S, Tallman MS. Enasidenib in mutant IDH2 relapsed or refractory acute myeloid leukemia. Blood. 2017;130 (6):722-31. https://doi.org/10.1182/blood-2017-04779405 .

231. Stoica GS, Greenberg HE, Rossoff LJ. Corticosteroid responsive fludarabine pulmonary toxicity. Am J Clin Oncol. 2002;25(4):340-1.

232. Stone RM, DeAngelo DJ, Klimek V, Galinsky I, Estey E, Nimer SD, Grandin W, Lebwohl D, Wang Y, Cohen P, Fox EA, Neuberg D, Clark J, Gilliland DG, Griffin JD. Patients with acute myeloid 
leukemia and an activating mutation in FLT3 respond to a small-molecule FLT3 tyrosine kinase inhibitor, PKC412. Blood. 2005;105(1):54-60. https://doi.org/ 10.1182/blood-2004-03-0891.

233. Sun Pharmaceutical Industries. Odomzo (sonidegib) [package insert]. Cranbury; 2017.

234. SuperGen. Mitozytrex (mitomycin) [package insert]. Dublin; 2002.

235. Swain SM, Ewer MS, Cortes J, Amadori D, Miles D, Knott A, Clark E, Benyunes MC, Ross G, Baselga J. Cardiac tolerability of pertuzumab plus trastuzumab plus docetaxel in patients with HER2positive metastatic breast cancer in CLEOPATRA: a randomized, double-blind, placebo-controlled phase III study. Oncologist. 2013;18(3):257-64. https://doi. org/10.1634/theoncologist.2012-0448.

236. Talpur R, Ward S, Apisarnthanarax N, BreuerMcham J, Duvic M. Optimizing bexarotene therapy for cutaneous T-cell lymphoma. J Am Acad Dermatol. 2002;47(5):672-84.

237. Tesaro Inc. Zejula (niraparib) [package insert]. Waltham; 2017.

238. Teva Pharmaceuticals Inc. Adrucil (fluorouracil injection) [package insert]. North Wales; 2017.

239. Teva Pharmaceuticals USA Inc. Bendeka (bendamustine hydrochloride) [package insert]. North Wales; 2017.

240. Thatcher N, Hirsch FR, Luft AV, Szczesna A, Ciuleanu TE, Dediu M, Ramlau R, Galiulin RK, Balint B, Losonczy G, Kazarnowicz A, Park K, Schumann C, Reck M, Depenbrock H, Nanda S, Kruljac-Letunic A, Kurek R, Paz-Ares L, Socinski MA. Necitumumab plus gemcitabine and cisplatin versus gemcitabine and cisplatin alone as first-line therapy in patients with stage IV squamous nonsmall-cell lung cancer (SQUIRE): an open-label, randomised, controlled phase 3 trial. Lancet Oncol. 2015;16(7):763-74. https://doi.org/10.1016/s14702045(15)00021-2.

241. Thornburg A, Abonour R, Smith P, Knox K, Twigg HL 3rd. Hypersensitivity pneumonitis-like syndrome associated with the use of lenalidomide. Chest. 2007;131 (5):1572-4. https://doi.org/10.1378/chest.06-1734.

242. Topp MS, Gokbuget N, Zugmaier G, Degenhard E, Goebeler ME, Klinger M, Neumann SA, Horst HA, Raff T, Viardot A, Stelljes M, Schaich M, KohneVolland R, Bruggemann M, Ottmann OG, Burmeister T, Baeuerle PA, Nagorsen D, Schmidt M, Einsele H, Riethmuller G, Kneba M, Hoelzer D, Kufer P, Bargou RC. Long-term followup of hematologic relapse-free survival in a phase 2 study of blinatumomab in patients with MRD in B-lineage all. Blood. 2012;120(26):5185-7. https:// doi.org/10.1182/blood-2012-07-441030.

243. Topp MS, Gokbuget N, Zugmaier G, Klappers P, Stelljes M, Neumann S, Viardot A, Marks R, Diedrich H, Faul C, Reichle A, Horst HA, Bruggemann M, Wessiepe D, Holland C, Alekar S, Mergen N, Einsele H, Hoelzer D, Bargou RC. Phase
II trial of the anti-CD19 bispecific T cell-engager blinatumomab shows hematologic and molecular remissions in patients with relapsed or refractory B-precursor acute lymphoblastic leukemia. J Clin Oncol. 2014;32(36):4134-40. https://doi.org/ $10.1200 /$ jco.2014.56.3247.

244. Umemura S, Yamane H, Suwaki T, Katoh T, Yano T, Shiote Y, Takigawa N, Kiura K, Kamei H. Interstitial lung disease associated with gemcitabine treatment in patients with non-small-cell lung cancer and pancreatic cancer. J Cancer Res Clin Oncol. 2011;137 (10):1469-75. https://doi.org/10.1007/s00432-0111013-1.

245. United Therapeutics Corp. Unituxin (dinutuximab) [package insert]. Silver Spring; 2015.

246. Vahid B, Marik PE. Infiltrative lung diseases: complications of novel antineoplastic agents in patients with hematological malignancies. Can Respir J. 2008;15 (4):211-6.

247. Valent P, Hadzijusufovic E, Schernthaner GH, Wolf D, Rea D, le Coutre P. Vascular safety issues in CML patients treated with BCR/ABL1 kinase inhibitors. Blood. 2015;125(6):901-6. https://doi.org/ 10.1182/blood-2014-09-594432.

248. Verweij J, van Zanten T, Souren T, Golding R, Pinedo HM. Prospective study on the dose relationship of mitomycin C-induced interstitial pneumonitis. Cancer. 1987;60(4):756-61.

249. Wadleigh M, Richardson PG, Zahrieh D, Lee SJ, Cutler C, Ho V, Alyea EP, Antin JH, Stone RM, Soiffer RJ, DeAngelo DJ. Prior gemtuzumab ozogamicin exposure significantly increases the risk of veno-occlusive disease in patients who undergo myeloablative allogeneic stem cell transplantation. Blood. 2003;102(5):1578-82. https://doi.org/ 10.1182/blood-2003-01-0255.

250. Wanchoo R, Abudayyeh A, Doshi M, Edeani A, Glezerman IG, Monga D, Rosner M, Jhaveri KD. Renal toxicities of novel agents used for treatment of multiple myeloma. Clin J Am Soc Nephrol. 2017;12 (1):176-89. https://doi.org/10.2215/cjn.06100616.

251. Wanchoo R, Jhaveri KD, Deray G, Launay-Vacher V. Renal effects of BRAF inhibitors: a systematic review by the cancer and the Kidney International Network. Clin Kidney J. 2016;9(2):245-51. https:// doi.org/10.1093/ckj/sfv149.

252. Waters MJ, Sukumaran S, Karapetis CS. PemetrexedInduced Interstitial pneumonitis: a case study and literature review. World J Oncol. 2014;5 (5-6):232-6. https://doi.org/10.14740/wjon845w.

253. Weiss RB, Poster DS, Penta JS. The nitrosoureas and pulmonary toxicity. Cancer Treat Rev. 1981;8 (2):111-25.

254. Wells SA Jr, Robinson BG, Gagel RF, Dralle H, Fagin JA, Santoro M, Baudin E, Elisei R, Jarzab B, Vasselli JR, Read J, Langmuir P, Ryan AJ, Schlumberger MJ. Vandetanib in patients with locally advanced or metastatic medullary thyroid cancer: a randomized, 
double-blind phase III trial. J Clin Oncol. 2012;30 (2):134 41. https://doi.org/10.1200/jco.2011.35.5040.

255. Welsh SJ, Corrie PG. Management of BRAF and MEK inhibitor toxicities in patients with metastatic melanoma. Ther Adv Med Oncol. 2015;7(2):122-36. https://doi.org/10.1177/1758834014566428.

256. Wenk KS, Pichard DC, Nasabzadeh T, Jang S, Venna SS. Vemurafenib-induced DRESS. JAMA Dermatol. 2013;149(10):1242-3. https://doi.org/10.1001/jamad ermatol.2013.5278.
257. West-ward Pharmaceuticals. Thiotepa [package insert]. Eatontown; 2015.

258. Zamorano JL, Lancellotti P, Munoz DR, Aboyans V, Asteggiano R, Galderisi M, Habib G, Lenihan DJ, Lip GY, Lyon AR, Fernandez TL, Mohty D, Piepoli MF, Tamargo J, Torbicki A, Suter TM. 2016 ESC position paper on cancer treatments and cardiovascular toxicity developed under the auspices of the ESC committee for practice guidelines. Kardiol Pol. 2016;74 (11):1193-233. https://doi.org/10.5603/kp.2016.0156. 